\title{
Methods in Cognitive Pupillometry: Design, Preprocessing, and Statistical Analysis
}

\author{
Sebastiaan Mathôt and Ana Vilotijević
}

Department of Psychology, University of Groningen, The Netherlands

Address for correspondence:

Sebastiaan Mathôt

Department of Psychology

University of Groningen

Grote Kruisstraat 2/1

9712TS Groningen

The Netherlands

Email: s.mathot@,rug.nl 


\begin{abstract}
Cognitive pupillometry is the measurement of pupil size to investigate cognitive processes such as attention, mental effort, working memory, and many others. Currently, there is no commonly agreed-upon methodology for conducting cognitive-pupillometry experiments, and approaches vary widely between research groups and even between different experiments from the same group. This lack of consensus makes it difficult to know which factors to consider when conducting a cognitive-pupillometry experiment. Here we provide a comprehensive, hands-on guide to methods in cognitive pupillometry, with a focus on trial-based experiments in which the measure of interest is the task-evoked pupil response to a stimulus. We cover all methodological aspects of cognitive pupillometry: experimental design; preprocessing of pupil-size data; and statistical techniques to deal with multiple comparisons when testing pupil-size data. In addition, we provide code and toolboxes (in Python) for preprocessing and statistical analysis, and we illustrate all aspects of the proposed workflow through an example experiment and example scripts.
\end{abstract}

Keywords: pupillometry, cognitive psychology, cognitive neuroscience, experimental design, data analysis 


\section{Methods in Cognitive Pupillometry: Design, Preprocessing, and Statistical Analysis}

The size of the eye's pupil reflects a wide range of cognitive processes (reviewed in Beatty \& Lucero-Wagoner, 2000; Loewenfeld, 1958; Mathôt, 2018). Increases in arousal or mental effort cause the pupil to dilate (i.e. become larger; Bradley et al., 2008; Hess \& Polt, 1960; Kahneman \& Beatty, 1966; Unsworth \& Robison, 2014); and increases in brightness cause the pupil to constrict (i.e. become smaller) even if the source of brightness is merely imagined (Laeng \& Sulutvedt, 2014), read about (Mathôt et al., 2017), covertly attended to (Binda et al., 2013a; Mathôt et al., 2013; Naber et al., 2013; Unsworth \& Robison, 2017), or maintained in visual working memory (Husta et al., 2019; Zokaei et al., 2019). The measurement of pupil size to investigate cognitive processes is what we will refer to in this article as cognitive pupillometry.

There is no commonly agreed-upon workflow for conducting cognitive-pupillometry experiments: design criteria, preprocessing steps, and statistical analyses all differ vastly between studies. Some attempts at standardization have been made, but these are not easily applicable to cognitive pupillometry because they are too broad (Kelbsch et al., 2019), focused on a different subfield (e.g., listening effort; Winn et al., 2018), or focused on specific preprocessing steps (Kret \& Sjak-Shie, 2018; Mathôt et al., 2018). Furthermore, guidelines are often conceptual rather than specific implementations, and the lack of concrete examples and code makes it difficult for researchers to incorporate these guidelines into their own workflow.

Here we aim to provide a hands-on, start-to-finish guide to cognitive pupillometry; that is, we provide concrete guidelines for designing cognitive-pupillometry experiments, for preprocessing of pupil-size data, and for conducting appropriate statistical analyses. All steps are illustrated with specific examples and code (in Python), which can be readily adapted to new experiments. In doing so, we will focus on what we consider to be a 'typical' cognitive-pupillometry 
bioRxiv preprint doi: https://doi org/10.1101/2022 02 23 481628; this version posted February 25,2022 The copyright holder for this preprint (which was not certified by peer review) is the author/funder, who has granted bioRxiv a license to display the preprint in perpetuity. It is made available under aCC-BY 4.0 International license.

experiment, which involves a computerized experiment with a trial-based structure in which the outcome of interest is a 'task-evoked pupil response' to a stimulus that is presented on each trial.

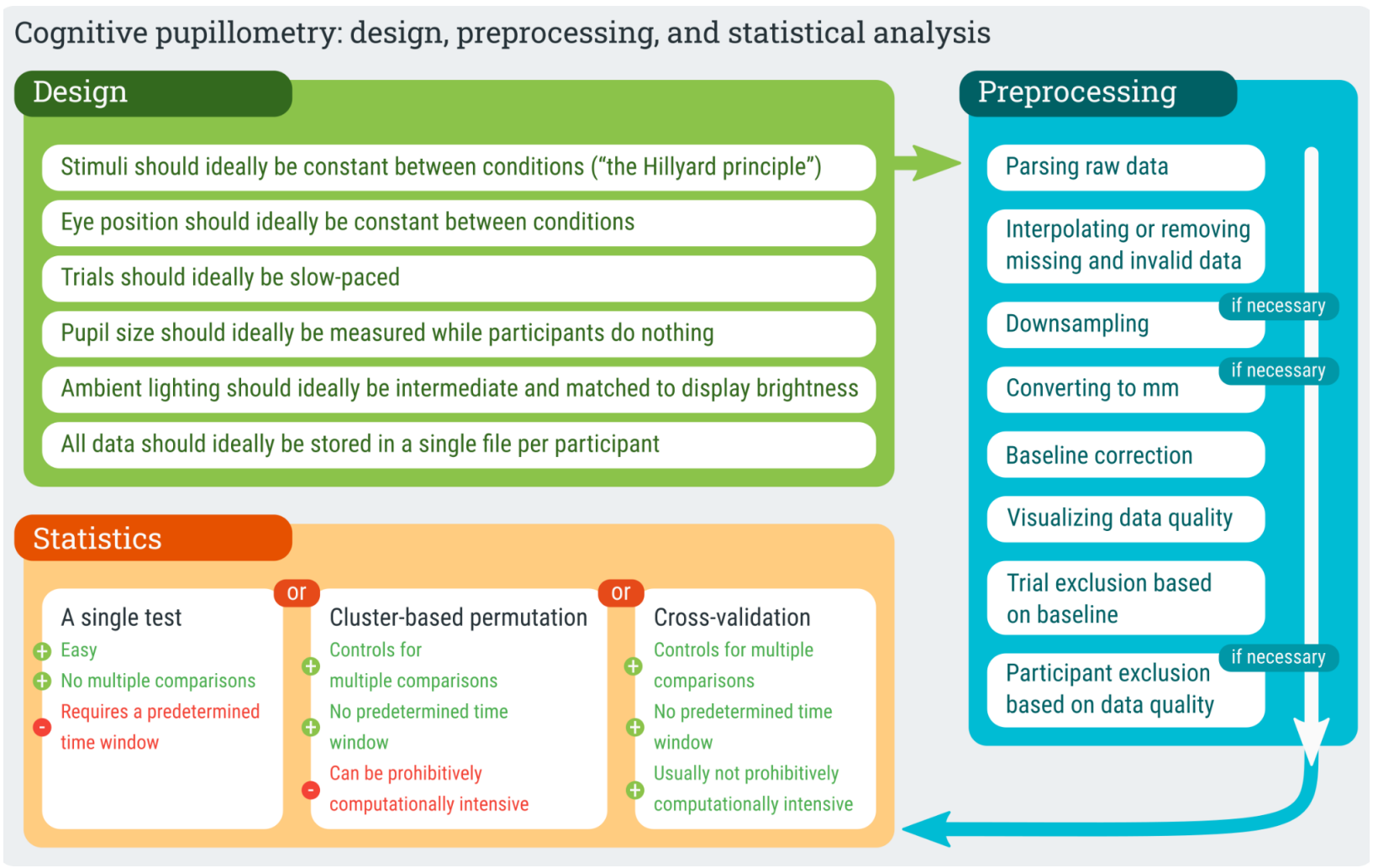

Figure 1. A schematic representation of the proposed workflow for conducting cognitive-pupillometry experiments.

Our guidelines are meant to be illustrative, rather than prescriptive; that is, we show how things could be done - rather than how they should be done - in order to implement a workflow that is both easy to implement and that meets contemporary standards for good scientific practice. Our guidelines are intended to be a starting point for researchers who are interested in adopting (part of) our workflow, either by re-using our code directly or by implementing (part of) our workflow using different tools (e.g. Geller et al., 2020; Hershman et al., 2019; Kinley \& Levy, 2021). 


\section{Example experiment}

Throughout this paper, we will refer to an experiment that we have recently conducted as an example. The theoretical motivation and methodological details for this experiment are described in Vilotijević and Mathôt (in prep.) and the associated preregistration (https://osf.io/ma4u9). Here, we will limit ourselves to those aspects of the experiment that generalize to many other experiments.

In brief, we asked whether pupil size increases as a function of attentional breadth; that is, we asked whether the pupil is larger when participants attend to the visual periphery as opposed to central vision. This question has been addressed several times before (Brocher et al., 2018; Daniels et al., 2012; Mathôt \& Ivanov, 2019); however, previous studies were all limited by differences in task difficulty or visual input between conditions. Therefore, we set out to carefully re-investigate this question while controlling for all possible confounding variables. 


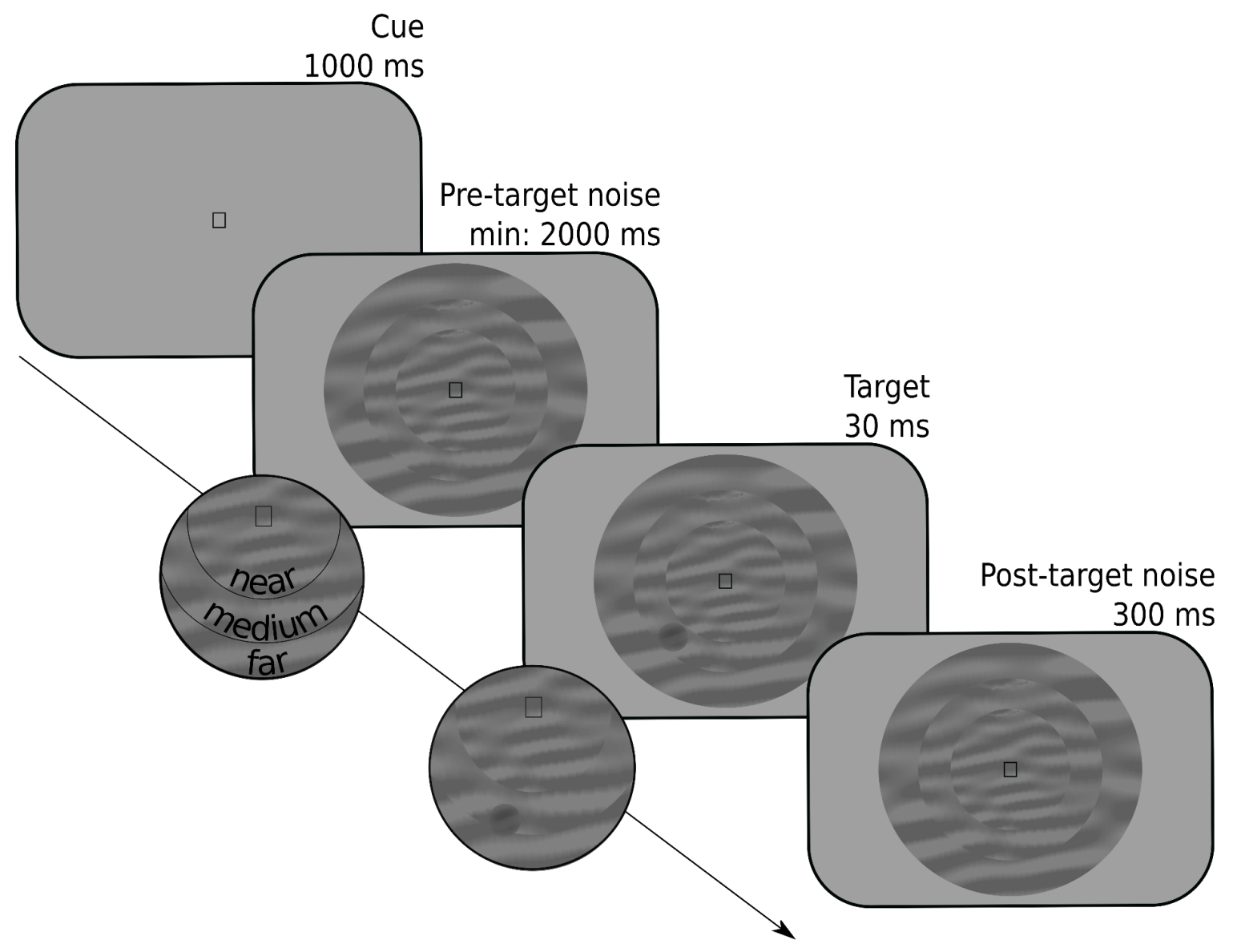

Figure 2. Schematic trial sequence for the example experiment. Participants reported whether a target was a luminance increment or, as in this example, a luminance decrement embedded in a dynamic stream of noise. The probable eccentricity (near, medium, far) of the target was indicated by a symbolic cue ( $a$ square in this example).

Each trial started with the presentation of a symbolic cue that informed participants of where a target was likely to appear: in a central ring (near eccentricity), in a ring around this central ring (medium eccentricity), or in an outer ring (far eccentricity). The mapping between cue symbol and eccentricity (e.g. $\square \rightarrow$ near, $\bigcirc \rightarrow$ medium, $\triangleleft \rightarrow$ far) was randomly varied between participants. Next, the three rings appeared; each ring was filled with dynamic noise, that is, patches of oriented noise that changed every $30 \mathrm{~Hz}$. At a random moment between 2000 and 
$3000 \mathrm{~ms}$ after the onset of the dynamic noise, a target (a subtle luminance increment or decrement) appeared, after which the dynamic noise continued for another $300 \mathrm{~ms}$. On $80 \%$ of the trials, the target appeared at a random location within the cued ring; on $20 \%$ of the trials, the target appeared at a random location within either of the other two rings. Participants indicated whether they had seen a luminance increment or decrement by pressing the left or right key on the keyboard. Accuracy was maintained at $66 \%$ by a two-up-one-down staircase that manipulated the opacity of the target, separately for each eccentricity, using only responses from validly cued trials.

Our primary measure of interest was pupil size in the $3000 \mathrm{~ms}$ interval after the presentation of the cue and before the earliest possible presentation of the target. We predicted that pupil size should increase with increased cue eccentricity. Based on previous studies that investigated the effect of shifts of attention on pupil size, we expected this effect to emerge at the earliest $750 \mathrm{~ms}$ after the presentation of the cue (e.g. Mathôt et al., 2013); however, we did not have an a priori expectation about at which moment during the remaining $2250 \mathrm{~ms}$ interval the effect should emerge, nor for how long the effect should persist.

\section{Experimental design}

A typical cognitive-pupillometry experiment follows the structure of a typical cognitive-psychology or cognitive-neuroscience experiment; that is, participants perform a computerized task that consists of many short trials during which stimuli are presented and responses are collected. By definition, one of the responses that is collected in a cognitive-pupillometry experiment is the pupil response; however, in many cases behavioral responses are collected as well. One or more independent variables are varied between trials. Our 
example experiment described above follows this structure. For a general introduction to experimental design for cognitive psychology and neuroscience, see Kingdom and Prins (2016). For the remainder of this article, we will assume that the reader is familiar with these kinds of experiments, and we will focus on those principles that are specific to cognitive-pupillometry experiments.

\section{Stimuli should ideally be constant between conditions}

Light is the main determinant of pupil size (Mathôt, 2018); therefore, when using visual stimuli, brightness should be constant between conditions. (Assuming, of course, that stimulus brightness is not itself a manipulation of interest.) For example, when comparing pupil dilation in response to arousing and non-arousing images, the average brightness of the arousing and non-arousing images should be the same (e.g. Bradley et al., 2008). This is a minimum requirement for cognitive-pupillometry experiments, and is widely understood by researchers.

However, even when brightness is strictly controlled, other low-level differences between stimuli may still affect pupil size, and these are much more difficult to control. There are-and this is unfortunate for cognitive pupillometrists - very few stimulus properties that do not affect pupil size: any form of visual change or movement induces pupil constriction (Barbur et al., 1992; Mathôt, Melmi, et al., 2015; Slooter \& van Norren, 1980; Ukai, 1985; Van de Kraats et al., 1977); color differences affect pupil size, generally such that blue stimuli result in a more sustained pupil constriction than red stimuli, though not necessarily in a more pronounced initial pupil constriction (Markwell et al., 2010); the distribution of brightness across the visual field affects pupil size, generally such that there is a bias towards foveal vision (Crawford, 1936; Hong et al., 2001), and to complicate matters further this foveal bias may be stronger for the 
initial phase of the pupil response than for more sustained pupil responses. And when it comes to auditory (Wang \& Munoz, 2015), tactile (van Hooijdonk et al., 2019), and other sensory modalities, increased stimulus intensity generally results in increased pupil size.

Because of the many, hard-to-predict ways in which low-level stimulus properties affect pupil size, stimuli should ideally be kept strictly constant between conditions such that only the cognitive state of the participant is varied. This is sometimes referred to as the Hillyard principle, after Steven Hillyard, who advocated this principle for event-related-potential (ERP) experiments (see also Luck, 2005).

Our example experiment abides by the Hillyard principle: there is no difference in the dynamic noise between conditions; and because the mapping between cue symbol and cued eccentricity is randomly varied between participants, even slight potential differences in the pupil response to the different symbols, such as a slightly more pronounced pupil constriction in response to a triangle than to a circle, do not confound our results. (The location and size of the target do differ between conditions; however, the target only appears after the interval of interest.)

However, some research questions inherently require experiments that deviate from the Hillyard principle; for example, investigating the difference in the pupil response to arousing and non-arousing images requires comparing two different sets of images with each other. Therefore, in such cases the Hillyard principle cannot be upheld; however, it can still be approximated by matching visual stimuli between conditions on as many low-level properties as possible, such as brightness (at least), visual saliency, and the distribution of luminance across the display (for examples, see Binda et al., 2013b; Naber \& Nakayama, 2013). Importantly, matching is always approximative and never perfect. Therefore, a key criterion is that a reasonable and informed 
person, such as a good peer reviewer, should agree that, while some confounds likely remain, they are unlikely to affect the results.

\section{Eye position should ideally be constant between conditions}

The position and movement of the eyes affect pupil size in two main ways. First, for most eye trackers, the angle from which the camera records the eye affects the recorded (as opposed to the actual) size of the pupil. This eye-position-based distortion of pupil size is often called the pupil-foreshortening error (PFE), and directly results from the fact that the surface of the pupil appears smaller in the camera image when it is recorded from the side, and thus looks like an ellipse, as compared to when it is recorded from the front, and thus looks like a circle. Some eye trackers are more susceptible to the PFE than others (Petersch \& Dierkes, 2021), and there are ways to compensate for the PFE algorithmically during data analysis (Brisson et al., 2013; Gagl et al., 2011; Hayes \& Petrov, 2016); however, although the PFE can certainly be minimized, you can never assume that pupil-size recordings are completely unaffected by the angle from which the eye tracker records the pupil.

Second, pupil size may really be affected by the position of the eye, independent of the PFE. This may happen when, say, the participant is seated such that it is more comfortable to look at the lower part of the display than at the upper part of the display (Mathôt, Melmi, et al., 2015). The increased effort or agitation associated with looking up may then result in pupil dilation. This is distinct from the PFE in the sense that it is not a recording artifact but a legitimate change in pupil size; however, even though it is real, it may still be a confounding factor in experiments in which eye position is not controlled. 
Third, eye movements are followed by pupil constriction (Knapen et al., 2016; Mathôt, Melmi, et al., 2015; Zuber et al., 1966), presumably as a result of the changes in visual input that are caused by eye movements. Constriction also occurs after eye blinks, which are similarly accompanied by a sudden change in visual input due to the closing and opening of the eyelid. This post-eye-movement and post-blink constriction can be compensated for algorithmically during data analysis (Knapen et al., 2016; Yoo et al., 2021); however, just as for the PFE, you can never assume that pupil size is completely unaffected by eye movements and blinks.

Because of these considerations, eye position should ideally be constant between conditions. In our example experiment, we ensured this by asking participants to keep their eyes fixated on a central stimulus throughout the experiment, thus minimizing eye movements. In some experiments in which it is necessary for participants to make eye movements, eye position can be strictly matched between conditions (Mathôt, Melmi, et al., 2015). However, in other experiments, for example experiments involving reading or other forms of natural eye movements, it is not feasible to strictly match eye position between conditions. In that case, eye position should be matched as closely as possible (Gagl et al., 2011). A key criterion is once again that a reasonable and informed person should agree that eye position is unlikely to affect the results.

\section{Trials should ideally be slow-paced}

The pupil light response (PLR) is the fastest pupil response with a latency of about $200 \mathrm{~ms}$; that is, the pupil starts to constrict around $200 \mathrm{~ms}$ after stimulus onset, and reaches maximal constriction around $750 \mathrm{~ms}$ after stimulus onset. Therefore, experimental manipulations that affect the strength and/or latency of the pupil light response to a stimulus tend to arise within 500 
ms (Binda \& Murray, 2015; Olmos-Solis et al., 2018; Wilschut \& Mathot, 2022). Another kind of pupil response that emerges rapidly is the orienting response that occurs when something captures attention; the orienting response is accompanied by a pupil dilation that peaks around 500 ms (Mathôt et al., 2014; Wang \& Munoz, 2014). Therefore, the oft-heard claim that pupil responses are very slow is not necessarily true.

However, what is true is that most cognitive-pupillometry experiments focus on pupil responses that do arise slowly; specifically, most experiments focus on pupil dilation as a marker of arousal or effort (e.g., Bradley et al., 2008; Rieger et al., 2015; Urai et al., 2017; Zekveld et al., 2010). If there is a clearly defined triggering stimulus, such as a burst of noise, pupil size tends to peak around 1 s after stimulus onset (Hoeks \& Levelt, 1993). However, in many studies, the trigger is an internal cognitive process without a clearly defined temporal onset, such as a challenging calculation (Hess \& Polt, 1964; Stoll et al., 2013) or a spoken sentence that is hard to understand (Koelewijn et al., 2012). In those cases, the resulting pattern of pupil dilation is hard to predict, but tends to be even slower and more diffuse.

Because of these considerations, cognitive-pupillometry experiments should ideally be slow-paced. However, experiments should not be too slow-paced for the simple reason that, as a researcher, you also need to be able to collect a sufficient amount of data within a reasonable time. As a rule of thumb, we suggest having the stimulus of interest followed by an interval of 2 - 3 s during which no other events happen (see also below), and which serves as the period of interest for pupil-size analysis. For this reason, there was at least $3000 \mathrm{~ms}$ between the onset of the cue and the onset of the target stimulus in our example experiment. 


\section{Pupil size should be ideally be measured while participants do nothing}

An increase in physical effort is accompanied by pupil dilation. This is true for intense physical exercise, and to a lesser-yet-measurable extent also for pressing a key on a keyboard, making a saccadic eye movements towards a target, pressing a button on a gamepad - or more generally any action through which participants respond in an experiment. Combined with the fact that response times (RTs) often vary between conditions, this response-locked pupil dilation can be a confounding factor when not properly accounted for.

To illustrate this issue, consider a hypothetical experiment in which participants indicate whether a character string corresponds to a word or not (i.e. a lexical-decision task); the difficulty of the word is the independent variable (difficult vs easy) and the pupil response to the word is the dependent variable. You might reasonably predict that processing a difficult word takes more mental effort, and should thus result in stronger pupil dilation, as compared to an easy word. (And given the proper experimental design this is quite likely what you would find.) However, participants also respond more slowly to difficult words than to easy words. Because of this difference in RT, you will likely find that the pupil initially dilates more strongly in response to easy as compared to difficult words - simply because of the response-locked pupil dilation.

Because of this, a task-evoked pupil response should not be recorded while participants are also giving a response. Rather, the response should be delayed until sometime after. In a lexical-decision experiment, this could mean that the character string is followed by a period of about 2 s of passive viewing during which pupil size is recorded; only after this period do participants indicate whether they saw a word or a non-word (for similar approaches, see Einhäuser et al., 2010; Snell et al., 2018). In our example experiment, we avoided 
response-locked pupil dilation by measuring pupil size during the interval preceding the target, before participants could even prepare a response, thus avoiding all motor preparation.

\section{Ambient lighting should ideally be intermediate and matched to display brightness}

Pupil size varies between roughly 2 and $8 \mathrm{~mm}$ in diameter (Mathôt, 2018; Pan et al., 2022). For most cognitive-pupillometry experiments, baseline pupil size should not approach these extremes, for the simple reason that maximally constricted or dilated pupils cannot constrict or dilate any further in response to experimental manipulations. Given that luminance is the main determinant of pupil size, experiments should therefore not be conducted in a very dark or very bright environment (unless there are specific reasons to do so, for example if the experiment requires dark-adapted pupils).

However, within a broad range of baseline pupil sizes, and thus for a broad range of ambient light levels, task-evoked pupil responses are reliably observed (Bradshaw, 1969; Cherng et al., 2020; Pan et al., 2022; Peysakhovich et al., 2017; Reilly et al., 2019; Steinhauer et al., 2004). As a rule of thumb, baseline pupil sizes between 3 and $7 \mathrm{~mm}$ are suitable. We conducted our example experiment in a dimly illuminated laboratory of $33 \operatorname{lux}^{1}$, which resulted in baseline pupil sizes of around $3.5 \mathrm{~mm}$. Some eye trackers only provide pupil size in arbitrary units without an easy way to convert these units to millimeters of diameter (see If necessary: converting pupil size from arbitrary units to millimeters of diameter); in this case, even without

\footnotetext{
${ }^{1}$ There are four main concepts that are all related to light but reflect different aspects. The total amount of light that is emitted by a light source, or luminous flux, is expressed in Lumen (or Candela); this measure is not often reported in experimental settings. The amount of light that falls from a light source onto a surface, or illumination, is expressed in Lux; this measure is often reported in experimental settings as an indication of the ambient light level in a laboratory. The amount of light that is emitted by a light source in a specific direction, or luminance, is expressed in Candela per square meter $\left(\mathrm{cd} / \mathrm{m}^{2}\right)$; this measure is often reported in experimental settings as an indication of the intensity of a visual stimulus. Finally, brightness refers to the subjective impression of luminance, and as such does not have a natural unit. Although luminance and brightness strictly speaking refer to different concepts, many authors, including ourselves on many occasions, use the terms interchangeably.
} 
knowing the exact pupil size of the participants, you can safely aim for a level of ambient light that is dim but still allows you to read comfortably.

It is also important to use a display luminance that roughly matches the luminance of the room; more specifically, using a bright stimulus display in an otherwise dark room results in discomfort glare, which is the unpleasant sensation that results from a small yet intense light source. Discomfort glare is accompanied by a pronounced decrease in pupil size (Tyukhova \& Waters, 2019). In our example experiment, we avoided discomfort glare by using a stimulus display of intermediate luminance $\left(33.1 \mathrm{~cd} / \mathrm{m}^{2}\right)$, which looked subjectively natural given the illumination of the room.

\section{All data should ideally be stored in a single file per participant}

A typical cognitive-pupillometry experiment is implemented using experiment-building software that is connected to an eye tracker that records gaze position and pupil size. In our example experiment, we used OpenSesame (Mathôt et al., 2012) to implement the experiment, PyGaze (Dalmaijer et al., 2014) to implement eye tracking in the experiment, and an EyeLink eye tracker to record pupil size. In set-ups of this kind, there are two distinct data files: the data file of the experiment-building software, in our case the OpenSesame . CSV file, which contains experimental variables, etc.; and the data file of the eye tracker, in our case the EyeLink . edf file, which contains eye-movement and pupil-size data. It is possible to cross-reference these two data files afterwards during data analysis; however, doing so is cumbersome and error-prone.

Therefore, it is good practice to log all data to a single file already during the experiment; usually, this single file is the eye-tracker data file, because it is more convenient to send time stamps, experimental variables, and other relevant information from the experiment-building 
software to the eye tracker, than it is to send all eye-position and pupil-size data from the eye tracker to the experiment-building software. In our example experiment, we did this by marking the start and end of relevant intervals (cue interval, dynamic-noise interval, etc.) in the EyeLink . edf file, and by logging all experimental variables (cue eccentricity, participant response, etc.) to the EyeLink . edf file at the end of each trial.

Finally, it is important to decide in advance how you intend to analyze the data (what analysis software you will use, what kind of information you need to extract, etc.), and to log the data in a format that makes this as easy as possible. In our example experiment, we used the Python EyeLinkParser module ${ }^{2}$; this module automatically processes variables that have been logged with var [name] [value] messages in the .edf file, and we therefore designed the experiment to send messages in this format. In contrast, had we intended to analyze the data with the EyeLink DataViewer software, we would instead have sent !V TRIAL_VAR [name] [value] messages, which is how DataViewer assumes that variables are logged.

\section{Preprocessing}

Here we use the term 'preprocessing' to refer to all steps involved in transforming the raw data, as it is stored during the experiment, into a format that is suitable for statistical analysis and visualization. The order matters, and the preprocessing steps below are described in the order in which they should be performed.

\footnotetext{
${ }^{2}$ https://github.com/smathot/python-evelinkparser
} 


\section{Parsing raw data into an analysis-friendly data structure}

Preprocessing starts with a set of raw data files, one for each participant, that contain all relevant data; this data includes experimental variables, start and end markers for trials and relevant intervals, and gaze-position and pupil-size data. In our example experiment, this data file is the EyeLink . edf file, but every brand of eye tracker uses its own custom format.

The first step is to parse the raw data into a data structure that is suitable for further processing. This generally involves a programming library or user interface that has been specifically designed for this purpose. For example, GazeR is an R library that parses raw eye-tracking data into an R data. frame object for further analysis (Geller et al., 2020). CHAP (Hershman et al., 2019) and PuPl (Kinley \& Levy, 2021) are MATLAB-based user interfaces that parse raw data into custom data structures that are used internally for further processing.

For our example experiment, we used the Python EyeLinkParser library to parse the raw data into a Python DataMatrix object ${ }^{3}$. Assuming that data has been logged following the assumptions of EyeLinkParser (see All data should ideally be stored in a single data file), the parser automatically recognizes experimental variables, and splits eye-position and pupil-size into separate intervals or 'epochs' (e.g. cue interval, dynamic-noise interval, etc.). This results in a spreadsheet-like data structure in which each row corresponds to a single trial, and each column corresponds to a single variable (Fig. 3). For variables that contain a single value per trial, such as response_time, one cell in the DataMatrix contains a single value. However, for variables that contain time series, such as pupil size during the cue interval (pupil_cue), one cell contains a series of values. You can think of this as a spreadsheet in

\footnotetext{
${ }^{3}$ https:/pydatamatrix.eu/
} 
which certain columns, those containing time series (SeriesColumn objects), stick out of the spreadsheet to accommodate their additional dimension: time.

The fact that time-series data (e.g. pupil size) can co-exist with single-value data (e.g. response time) in a single data structure is convenient for further analysis.

\begin{tabular}{|ccccc} 
Trials & response_time & cue_eccentricity & subject_nr & pupil_stream \\
$\qquad 732$ & medium & 1 & {$[0.00,0.03,0.01, \ldots,-0.73]$} \\
1112 & far & 1 & {$[0.00,0.01,0.00, \ldots, 0.16]$} \\
888 & near & 1 & {$[0.00,-0.03,-0.05, \ldots,-1.11]$} \\
$\vdots$ & $\vdots$ & $\vdots$ & $\vdots$ & \\
2604 & medium & 30 & {$[0.00,0.02,0.02, \ldots, 0.44]$}
\end{tabular}

Figure 3. A schematic representation of a DataMatrix object. Each row represents a trial. Each column represents a variable. Pupil-size data (here: pupi___stream) is stored in a special column type (SeriesColumn) that has an additional dimension to store how pupil size changes as a function of time (sample number).

Interpolating or removing missing and invalid data (e.g. due to blinks)

Missing data occurs when the eye tracker fails to record any pupil size at all. This can happen when the participant moves slightly, causing the eye tracker to lose the eye, when there is a technical malfunction of the eye tracker, or when the participant has fully closed the eyes. Most eye trackers indicate missing data with a special value; in the case of the EyeLink, missing data is initially indicated with the value 0 . During parsing, missing data should ideally be recoded to $\mathrm{NaN}$ values (not a number), which is a standard way to represent missing or invalid data. 
Invalid data occurs when the eye tracker does record pupil size, but the measured pupil size does not accurately reflect the actual pupil size. This can happen when the participant is in the process of blinking, such that the pupil is partly obscured by the eyelid, or when the eye tracker does not reliably extract the entire pupil from the camera image. Unlike missing data, invalid data is not represented in any special format, and can only be detected indirectly, through various quality-check measures.

Pupil-size data, even when it is of high quality, invariably contains missing and invalid data (discussed also in Kret \& Sjak-Shie, 2018; Mathôt et al., 2018). Therefore, an important step in preprocessing is to first identify invalid data and remove it, that is, to turn invalid data into missing data. In principle, this is sufficient, because missing data does not cause measurement error in the way that invalid data does. However, missing data does reduce statistical power, and can make visualizations of average pupil data look less smooth. For these reasons, it is good practice to interpolate missing data whenever this is reasonably possible.

The goal of interpolation is to estimate missing or invalid data by drawing a line through valid data points. This is usually done using either a linear interpolation, which draws a straight line between two valid data points, or using quadratic (cubic-spline) interpolation, which draws a smooth line through four valid data points, which results in interpolations that more closely resemble natural pupil-size changes. There are various ways in which interpolation of missing pupil-size data can be implemented; such procedures are sometimes referred to as 'blink reconstruction', since blinks are the primary reason for missing data (for alternative implementations, see Hershman et al., 2019; Kinley \& Levy, 2021; Kret \& Sjak-Shie, 2018). Here we will focus on the 'advanced' algorithm as implemented in the DataMatrix blinkreconstruct () function. This algorithm is based on the procedure described in 
Mathôt (2013), and has been optimized to catch many of the edge cases in which the original algorithm failed.

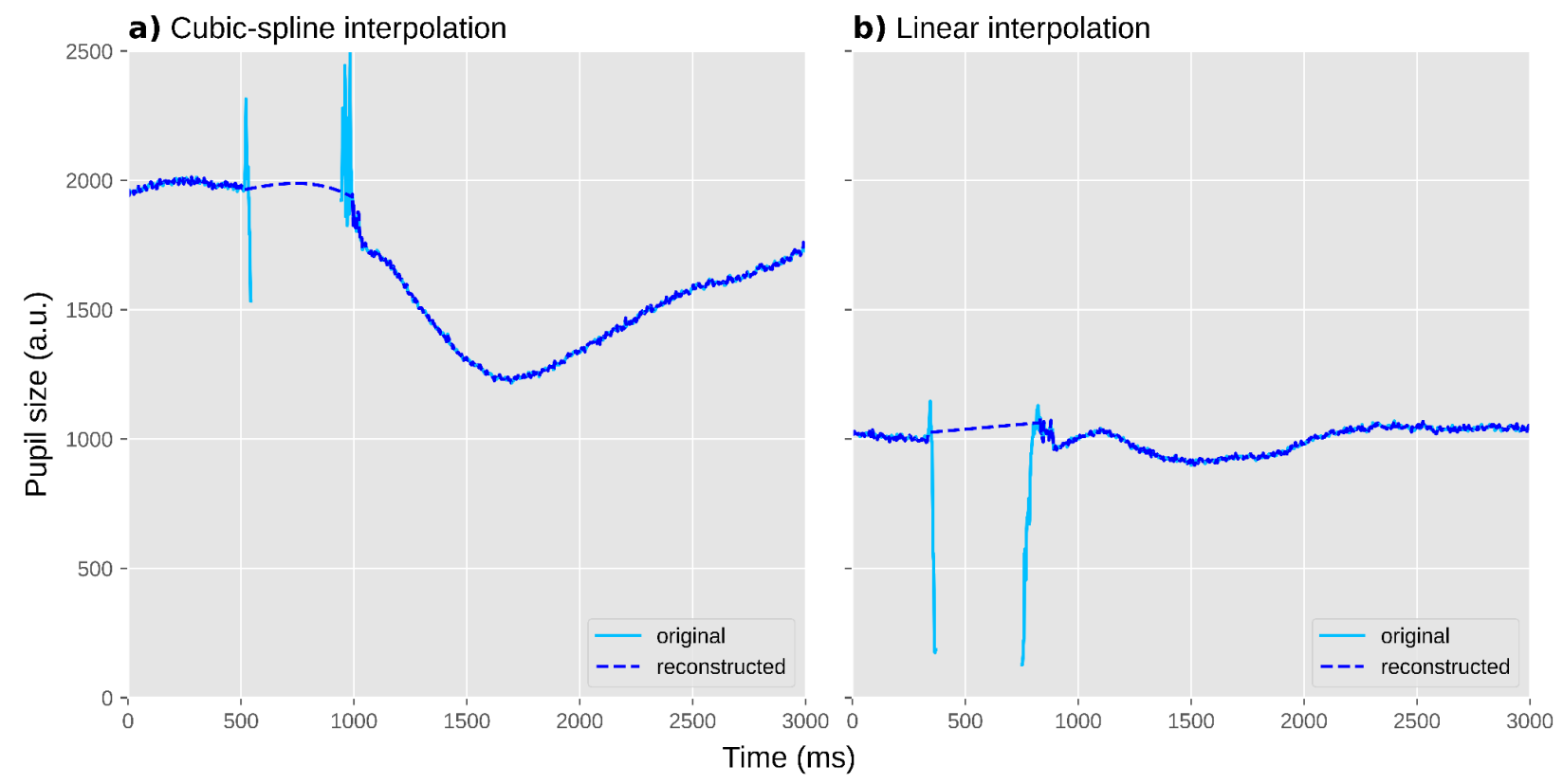

Figure 4. Two individual trials during which a blink occurred. Blinks are often characterized by a sharp drop in pupil size (due to closing of the eyelid) followed by a period of missing data (due to the eyelid being fully closed), followed in turn by sharp increase in pupil size (due to the eyelid re-opening). a) An example of cubic-spline interpolation, based on four points around the blink. This results in a smooth curve. b) An example of linear interpolation, based on two points around the blink. This results in a straight line.

The algorithm uses a recursive procedure in which it first reconstructs pupil size during blinks. To do so, it identifies the onset and offset of a blink based on a velocity threshold; that is, a blink is assumed to start when pupil size rapidly decreases (due to closing of the eyelid, thus rapidly obscuring the pupil), and to end when pupil size stabilizes again after a period of rapid pupil-size increase (due to opening of the eyelid). A blink is generally preceded and followed by several milliseconds of unreliable data; therefore, a margin (by default $10 \mathrm{~ms}$ ) around the blink is also 
marked as missing. A blink cannot be longer than a certain duration (by default $500 \mathrm{~ms}$ ); longer blink-like periods are not reconstructed, based on the intuition that interpolation only makes sense for brief periods during which pupil size is predictable.

Once the onset and offset of a blink have been determined, the algorithm first tries to perform cubic-spline interpolation; this requires two additional points, equally spaced before the onset of the blink and after the offset of the blink. If these points can be determined, missing data during the blink is interpolated with a smooth curve (Fig. 4a). If these points cannot be determined, for example because one of the points falls outside the time series or inside another blink, the blink is interpolated linearly with a straight line (Fig. 4b).

Once a blink has been identified and reconstructed, the algorithm starts again from scratch (i.e. it is a recursive procedure) until no blinks are identified anymore. At this point, any remaining invalid data is marked as missing. This is done by removing all pupil-size data points that deviate more than a threshold from the mean pupil size of the series (by default $\pm 3 \mathrm{SD}$ ) or where pupil size (very) rapidly increases or decreases. Finally, margins (by default $20 \mathrm{~ms}$ ) around all periods of missing data are similarly marked as missing.

\section{If necessary: downsampling}

The latency of the pupil light response is around $200 \mathrm{~ms}$, and the earliest cognitive effects on pupil size emerge around $500 \mathrm{~ms}$ after the triggering stimulus (see Trials should ideally be slow-paced). This means that a sampling rate of $20 \mathrm{~Hz}$, such that pupil size is measured once every $50 \mathrm{~ms}$, is sufficient when, as in most cognitive-pupillometry experiments, you are interested in how pupil size differs between experimental conditions. (A higher sampling rate is desirable when you are interested in measures such as maximum constriction velocity, which 
requires many measurements during the fast initial constriction of the pupil light response.) For visualization purposes, a higher sampling rate is desirable to be able to generate smooth figures of pupil size over time; however, even for visualization, a sampling rate of more than $100 \mathrm{~Hz}$ is rarely useful.

Therefore, if you are using an eye tracker that records at a higher sampling rate, it is convenient to downsample the signal to $100 \mathrm{~Hz}$. The main reason for doing so is to reduce memory consumption, which without downsampling can easily become prohibitive for large datasets. In the case of our example experiment, we downsampled the original $1000 \mathrm{~Hz}$ recording by a factor of 10 to $100 \mathrm{~Hz}$. Importantly, downsampling should be performed after preprocessing steps that benefit from a high sampling rate, such as blink reconstruction.

If necessary: converting pupil size from arbitrary units to millimeters of diameter

For most cognitive-pupillometry experiments, the measure of interest is not the size of the pupil in absolute terms, but rather the change in pupil size between conditions. As such, the unit in which pupil size is reported is not crucially important. Nevertheless, many researchers prefer to express pupil size in millimeters of diameter. Doing so makes it possible to verify that pupil size does not approach physiological limits (see Ambient lighting should ideally be intermediate and matched to display brightness), and makes it possible to compare effect sizes in natural units: a pupil constriction of $4 \mathrm{~mm}$ in response to a flash of light is in an entirely different class of effect size than a pupil dilation of $0.1 \mathrm{~mm}$ in response to a challenging calculation, even though both may be highly significant when tested statistically.

Some eye trackers, such as the Tobii series of eye trackers, automatically report pupil size in millimeters; they are able to do so because the eye tracker contains a depth sensor that estimates 
the distance between the eye tracker and the participant's face, which in turn allows the eye-tracking software to transform pupil size from camera-image-specific units (e.g. pixel count, or the size of the longest axis of the best fitting ellipse in pixels) to millimeters. However, many eye trackers, such as the EyeLink, do not offer this functionality and simply express pupil size in arbitrary units. In that case, it may be possible to determine a conversion formula yourself.

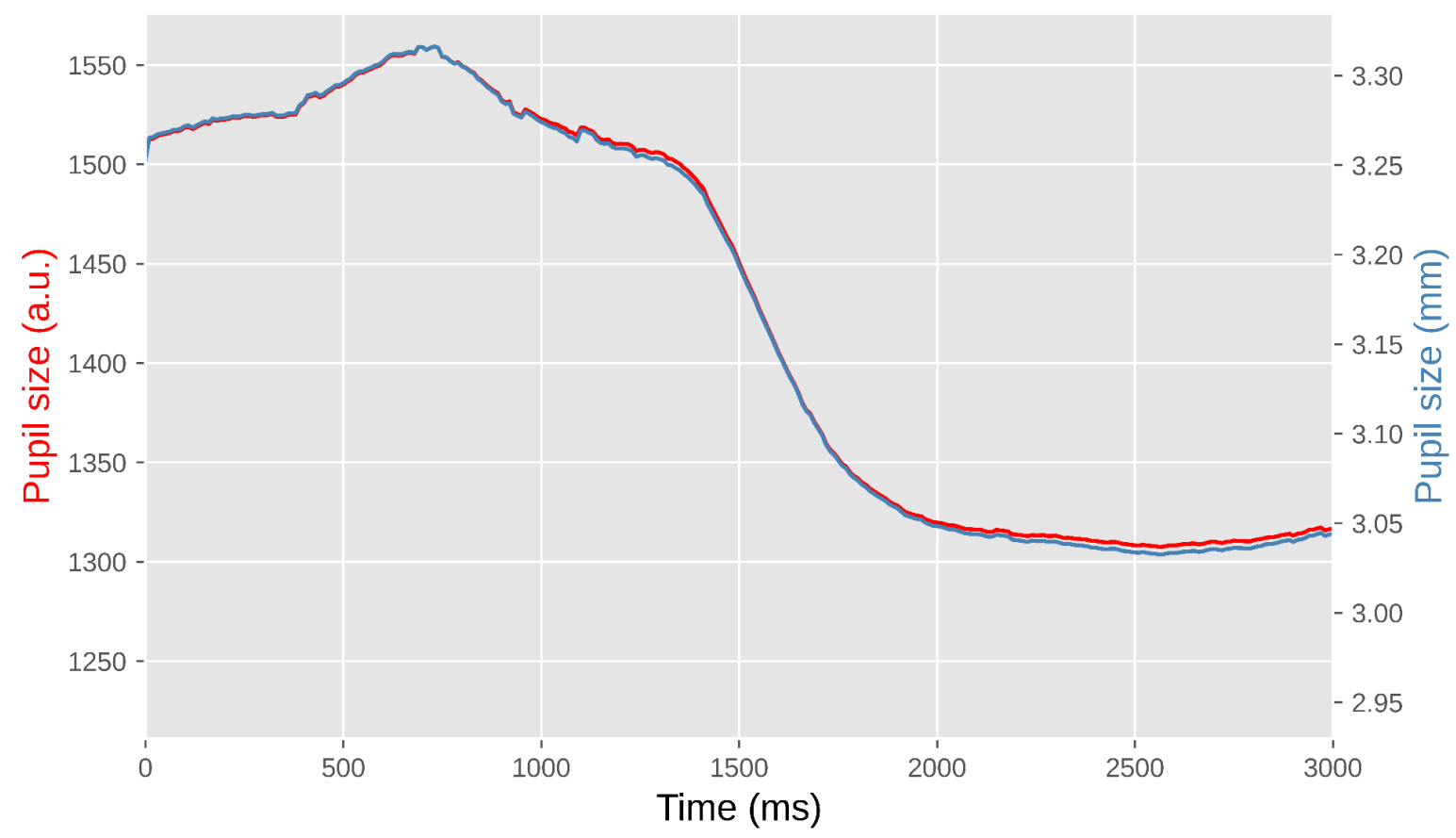

Figure 5. Pupil size during a single trial from our example experiment. The left y-axis shows pupil size in arbitrary units as recorded by the EyeLink eye tracker. The right y-axis shows pupil size in millimeters of diameter based on a conversion formula. The lines do not overlap perfectly because the conversion is not linear.

In the case of our example experiment, our group had previously determined a conversion formula for our laboratory set-up, allowing us to convert the EyeLink's arbitrary units to millimeters (Wilschut \& Mathot, 2022). To do so, we printed fifteen black circles of various 
known sizes on a white sheet of paper and held this in front of the eye tracker just above the chinrest. The EyeLink accepts these circles as pupils, and reports a size for them. This provided us with ground-truth pupil sizes (i.e. the size of the circles as measured with a ruler) and recorded pupil sizes in arbitrary units, from which we derived a conversion formula with the help of an online application that automatically determines the best-fitting function given a set of predictors and observations.

\section{Baseline correction}

Pupil size fluctuates in waves of several seconds that reflect the waxing and waning of arousal (Mathôt, Siebold, et al., 2015; Reimer et al., 2014), and in slower waves that reflect more prolonged states of fatigue and wakefulness (Geacintov \& Peavler, 1974; Lowenstein et al., 1963). In cognitive-pupillometry experiments that are not primarily concerned with general states of arousal and wakefulness, such fluctuations are a source of noise that reduce statistical power to detect differences in task-evoked pupil responses between conditions.

Baseline correction is a technique to remove the impact of trial-to-trial fluctuations in pupil size (Mathôt et al., 2018). This is done for each trial separately, usually by subtracting the mean pupil size during a 'baseline period' from all subsequent pupil-size measurements (subtractive baseline correction; recommended), or sometimes by dividing all subsequent pupil-size measurements by the mean baseline pupil size (divisive baseline correction; not recommended, see Mathôt et al., 2018). As a result, pupil size starts from 0 (for subtractive correction) or 1 (for divisive correction) during the baseline period on every trial, and only the change in pupil size-the task-evoked pupil response-remains. 
Crucially, a baseline period should itself not be affected by the experimental manipulations. In practice, this means that the baseline period should come before the stimulus that triggers the task-evoked pupil response; alternatively, the baseline period can coincide with the onset of the triggering stimulus, as long as the duration of the baseline period is below the minimum latency of the pupil response (i.e. less than $200 \mathrm{~ms}$ ). Also, the baseline period should not contain too many blinks or other artifacts that could affect baseline pupil size (see Excluding trials based on baseline pupil size); therefore, we prefer to use a short baseline period, since this reduces the chance of artifacts. In our example experiment, we used the first $50 \mathrm{~ms}$ after the onset of the cue as the baseline period, because the cue is the stimulus that triggers the shift in attentional breadth that we hypothesized would affect pupil size. 


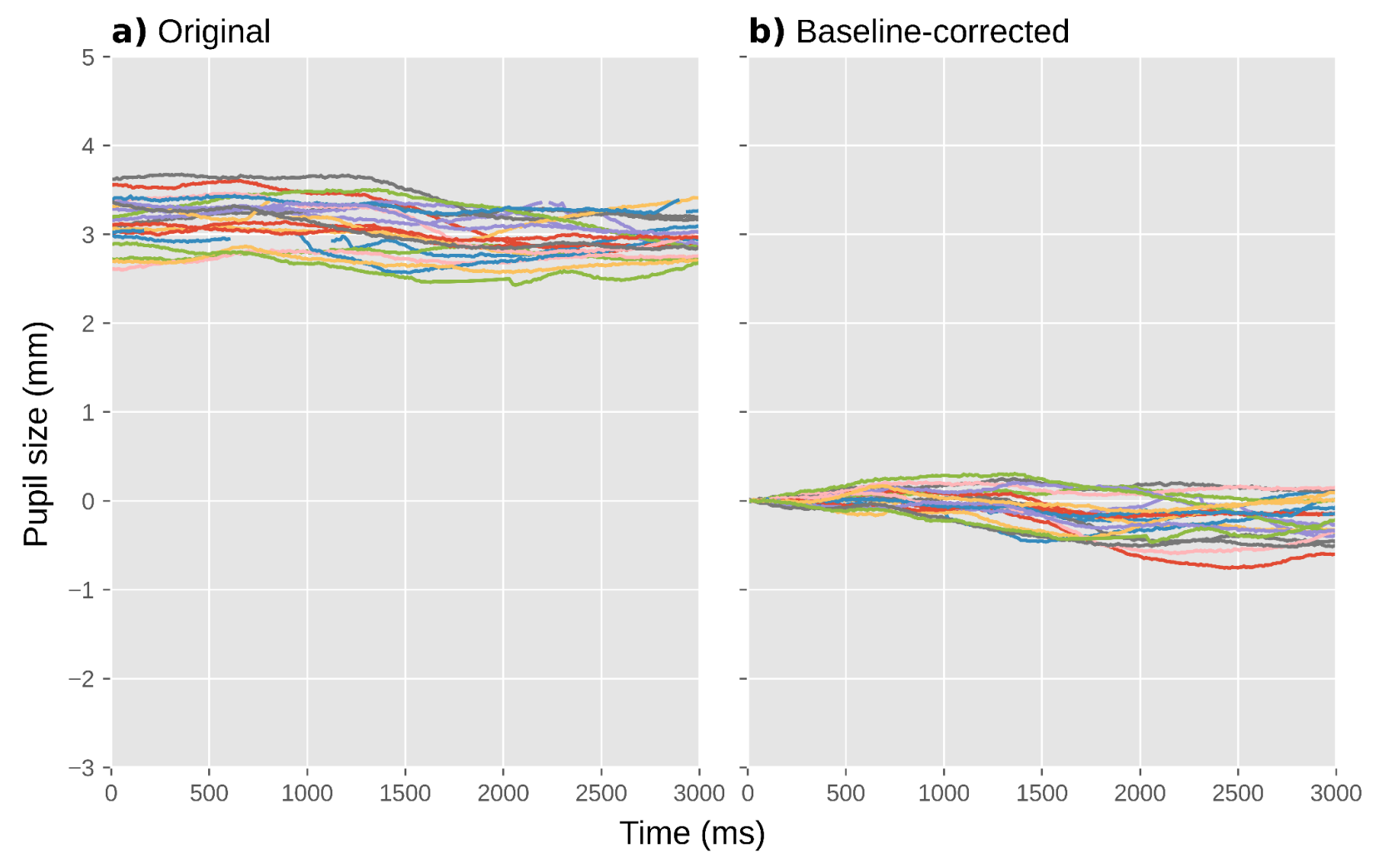

Figure 6. Pupil size during a selection of individual trials from our example experiment. a) Before baseline correction. b) After baseline correction.

\section{Verifying and visualizing data quality}

In an ideal world, pupil-size data consists of smooth traces that are unaffected by blinks, eye movements, or recording artifacts. In addition, in this ideal world, gaze position is perfectly constant while pupil size is being recorded. However, in the real world, the quality of pupil-size data never attains this ideal. Therefore, it is important to get a sense of the quality of your dataset by visualizing relevant aspects of the data.

A useful visualization is to plot all pupil-size traces as semi-transparent lines in a single figure (Fig. 7a); good-quality data will look like a tangle of lines. Another useful visualization is a histogram of baseline pupil sizes (Fig. 7b); good-quality data will be roughly normally 
distributed, and often has a slight skew that can either be to the left (as in Fig. 7b; less common) or to the right (more common). These figures are best created separately for each participant to avoid clutter, and collapsed across experimental conditions to avoid yourself from being biased by whether the data show the desired effect when assessing data quality.
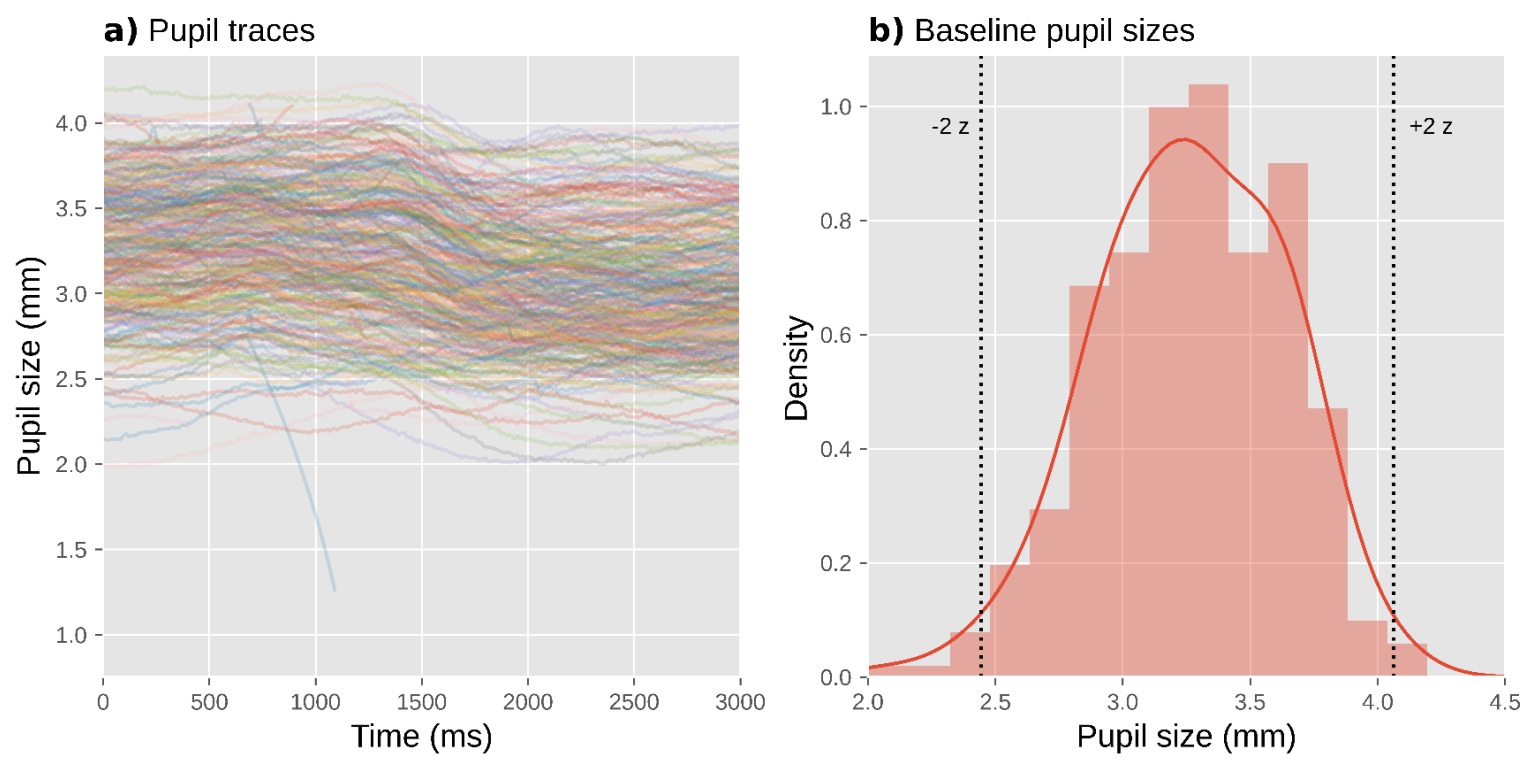

Figure 7. Visualization of data quality for a single participant from our example experiment. a) A plot with pupil size during all trials plotted as semi-transparent lines allows you to check visually for distortions, such as downward spikes. b) A histogram of baseline pupil sizes allows you to check visually for clusters of outliers.

A key sign of poor data quality is the presence of spikes that shoot downwards (but rarely upwards) from this tangle of lines: these spikes correspond to blinks or other recording artifacts that were not successfully interpolated or removed. A handful of such spikes can occur even in high-quality data (such as the downwards spike around $1000 \mathrm{~ms}$ in Fig. 7a), and this is not necessarily a cause for concern; however, if there are too many spikes, this adds substantial variability to the data, thus decreasing statistical power. What constitutes 'too many' is largely a 
matter of experience and tolerance, but a rule of thumb is that no more than $5 \%$ of trials should show such spikes. If there are more spikes, then it is worthwhile to reconsider the procedure for interpolating and removing invalid data (see Interpolating or removing invalid data), for example by optimizing the blink-reconstruction parameters for the sampling rate of the eye tracker.

Another sign of poor data quality is the presence of lines that are far above (but rarely below) the others, or that start from zero but then quickly $(<200 \mathrm{~ms})$ shoot upwards: these lines correspond to trials on which there were artifacts during the baseline period, resulting in extremely small baseline pupil sizes (which should also be evident in the histogram of baseline pupil sizes) and thus extremely large baseline-corrected pupil sizes (Mathôt et al., 2018). Such trials add substantial variability to the data, thus decreasing statistical power. Fortunately, it is straightforward to identify and exclude such trials (see Trial exclusion based on baseline pupil size). 


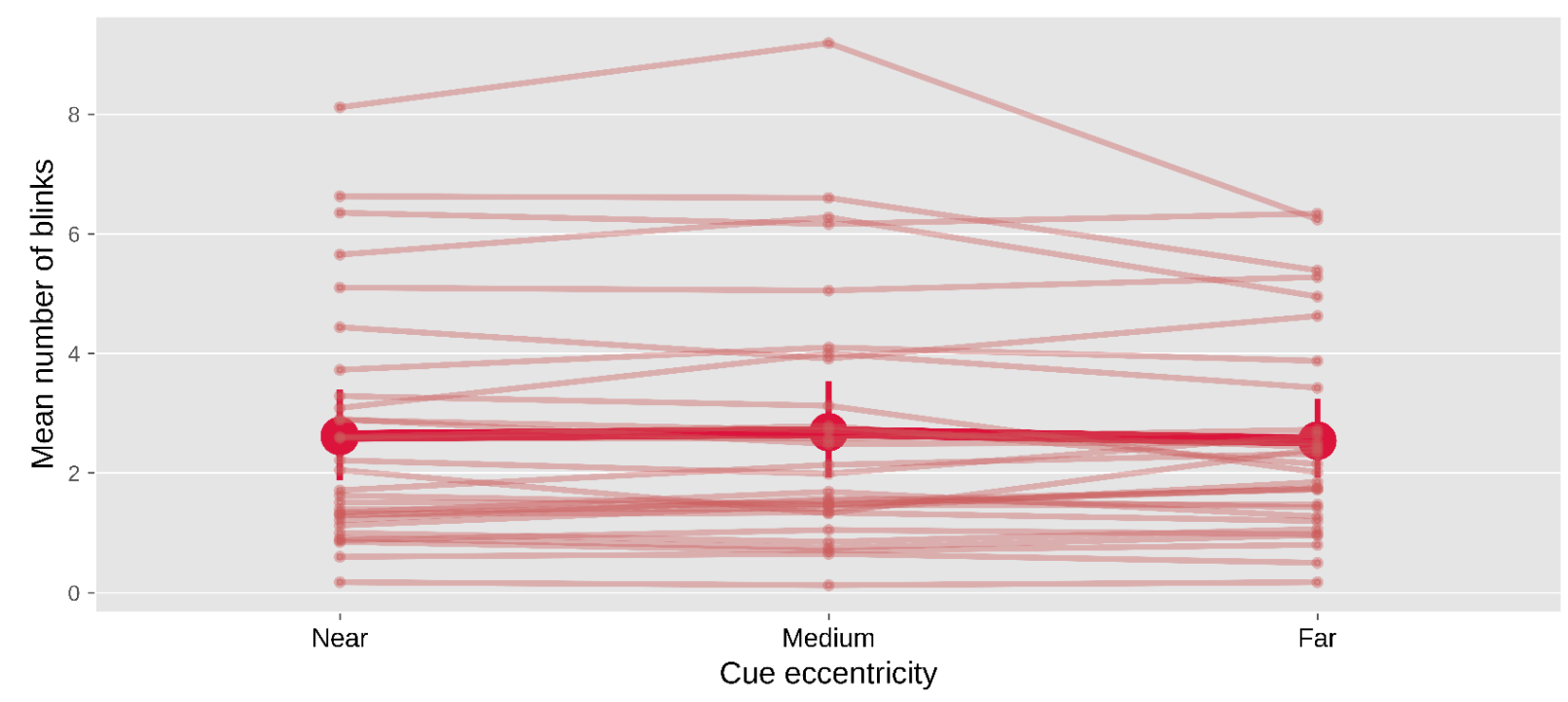

Figure 8. A plot with blink rate as a function of experimental condition and participant allows you to check visually for systematic differences in blink rate between conditions. Error bars indicate 95\% between-subject bootstrapped confidence intervals. Based on complete data from our example experiment.

A third useful visualization is to plot the average number of blinks per trial, separately for each experimental condition and participant (Fig. 8). Participants differ considerably in how often they blink; in our example experiment, some participants rarely blink at all, while others blink several times on each trial. This is not in itself a problem; however, blink rate should not systematically differ between experimental conditions. If it does, then this is inherently problematic when interpreting differences in pupil size between experimental conditions, because pupil size is affected by blinks (Yoo et al., 2021). If you find differences in blink rate, then you may want to reconsider the experimental design in order to avoid this. If changing the design is not possible, then differences in blink rate should at least be transparently reported, 
such that readers and reviewers can assess for themselves whether these differences are likely to confound the results.

Some eye trackers, such as the EyeLink that we used for our example experiment, automatically detect blinks during recording, in which case it is easy to create a plot like Fig. 8. For eye trackers that do not automatically detect blinks, you can use a custom blink-detection algorithm (e.g. Hershman et al., 2018). Alternatively, you can count the number of missing data points on each trial as an easy proxy for the number and duration of blinks (although of course missing data can also result from other factors, such as recording errors).

In our example experiment, there was no notable difference in blink rate between experimental conditions (Fig. 8), although there were substantial differences in blink rate between participants.

A fourth and final useful visualization is to show gaze position over time. In our example experiment, participants were instructed to keep their eyes fixated on a central fixation dot; therefore, the most relevant measure of eye position is a deviation from the display center in any direction. To visualize this, we plotted the absolute difference between the $\mathrm{x}$ coordinate and the horizontal display center, over time and averaged across trials but separately for each experimental condition, in one panel (Fig. 9a), and the absolute difference between the y coordinate and the vertical display center in another panel (Fig. 9b). 


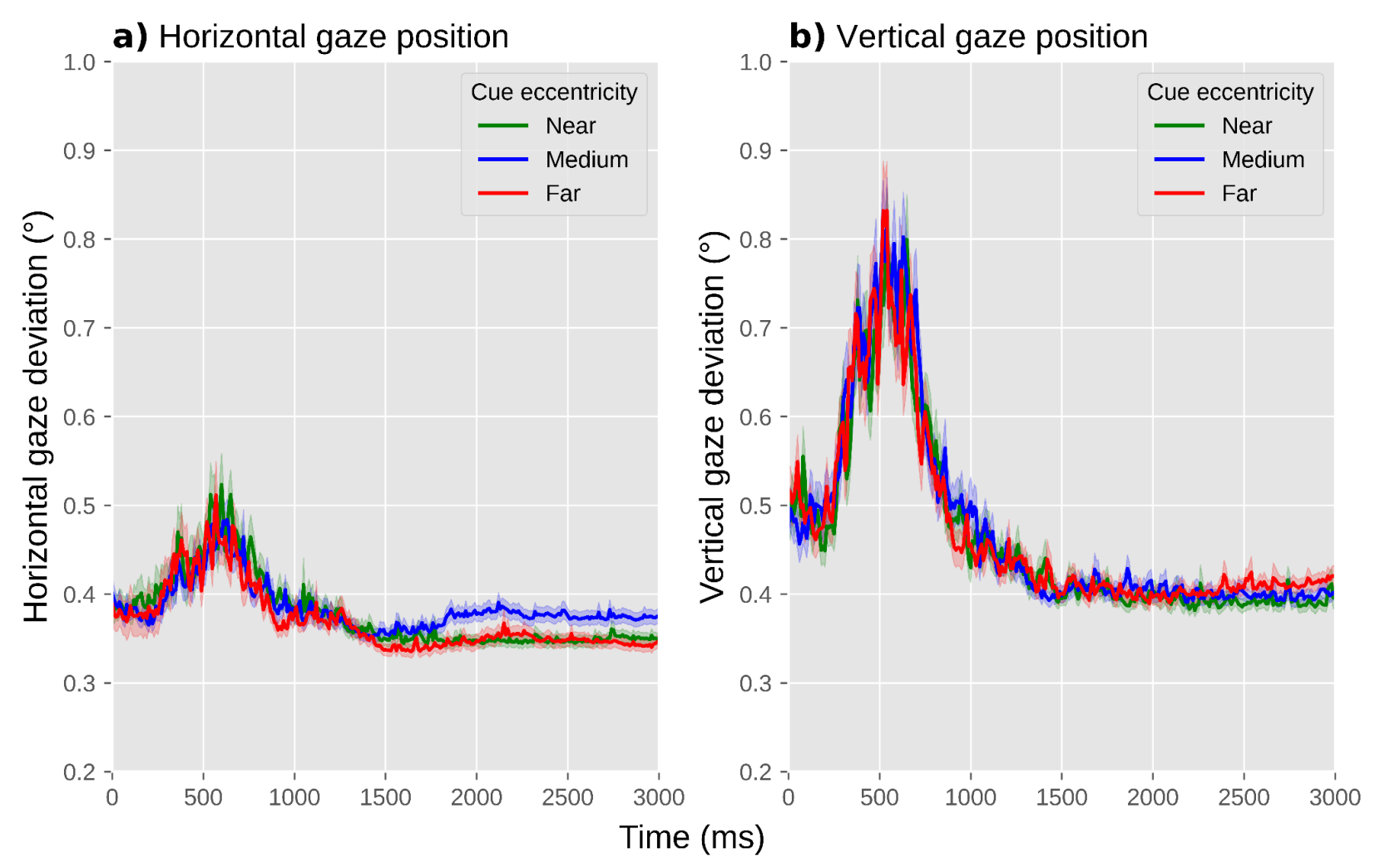

Figure 9. A plot with the deviation of horizontal (a) and vertical (b) eye position from the display center as a function of experimental condition allows you to visually check whether there are systematic differences in gaze deviation between conditions. Error shadings indicate grand standard error. Based on complete data from our example experiment.

When assessing differences in gaze position between experimental conditions, the situation is much the same as for blink rate: ideally, there should not be any systematic differences between experimental conditions in either horizontal or vertical gaze position; if there are, this may require a change to the experimental design, and if this is not possible, then these differences should be reported.

In our example experiment, there was no notable difference between conditions in absolute gaze deviation from the center (Fig. 9). Gaze deviation briefly increased around $500 \mathrm{~ms}$ after the onset 
of the cue, reaching a maximum deviation of about $0.5^{\circ}$ for horizontal and $0.8^{\circ}$ for vertical eye position, but again this occurred in all conditions.

\section{Excluding trials based on baseline pupil size}

As described above, blinks and recording artifacts during the baseline period may result in very small baseline pupil sizes (see Baseline correction); in turn, this results in very large baseline-corrected pupil sizes, which add variability to the data and may substantially reduce statistical power (Mathôt et al., 2018). Therefore, trials with extreme baseline pupil sizes should be excluded from analysis.

To do so, you can first convert baseline pupil sizes to $z$-scores. This should be done separately for each participant, because what is an extremely small pupil size for one participant may be a fairly normal pupil size for another participant. Next, all trials where the $z$-scored baseline pupil size is larger than 2 or smaller than -2 are excluded (indicated by the vertical lines in Fig. $7 b$ ). This simple, predetermined criterion tends to effectively remove problematic trials. In the data from our example experiment, we excluded 402 trials (5.58\%) based on this criterion.

\section{If necessary: excluding participants based on data quality}

Data quality can differ substantially between participants, for example because of contact lenses, glasses, eye make-up, or other factors that reduce the ability of the eye tracker to record the pupil. In rare cases, this may be a reason to exclude a participant's data from analysis altogether. Ideally, exclusion criteria should be specified in advance; however, because of the many hard-to-predict ways in which the quality of pupil-size data can be poor, you may still encounter 
participants that warrant exclusion for reasons that you did not foresee. Therefore, it is crucial to transparently report how many participants were excluded and why.

Fortunately, a few precautionary steps often avoid having to exclude any participants. First, make sure to properly set-up the participant before the experiment. For example, make sure that the participant is well-positioned in front of the eye tracker, that the chin rest is comfortable, that the focus of the eye-tracking camera is adjusted (if applicable), that the eye tracker is well-calibrated, etc. Second, abort an experimental session if you suspect that the data will be unusable, for example because the eye tracker regularly fails to track the eye. By not collecting a complete dataset, you prevent yourself from having to decide post-hoc-and thus biased by whether you like the results-whether to include the data or not. (Assuming, of course, that incomplete data is never included, which is a general rule that most researchers, including ourselves, follow unless there is a good reason to do otherwise.) When aborting a session, consider that participants may take this as a sign of failure on their part, and it is therefore courteous to explain to them that eye tracking is prone to technical issues even if participants perform well. Third, use a statistical technique that is able to deal with large differences in observations between participants; that way, you do not have to exclude participants if a substantial number of trials is excluded due to, for example, extremely small baseline values (see Excluding trials based on baseline pupil size).

In our example experiment, we did not exclude any participants from analysis.

\section{Visualization and statistical analysis}

From a statistical perspective, most cognitive-pupillometry experiments are interested in the following question: do any of my experimental manipulations affect pupil size at some moment 
during an interval of interest? This is also the case for our example experiment, in which we were interested in whether cue eccentricity affects pupil size at some moment between the presentation of the cue and the target. (For different statistical techniques to address different research questions see e.g. Fink et al., 2021; Reilly et al., 2021).

\section{Multiple comparisons in analysis of pupil-size data}

For our purpose, the main factor that complicates analysis of pupil-size data, as compared to response-time and most other behavioral data, is that pupil size is a continuous signal or 'time series'; that is, the data does not contain a single dependent variable, but rather a series of dependent variables, one for each time sample, that reflect how pupil size changes over time.

In our example experiment, there were 300 such values, one for each $10 \mathrm{~ms}$ of our $3000 \mathrm{~ms}$ cue-target interval. This means that we could, in principle, conduct 300 statistical tests, one for each sample, and then see if one of these tests is 'significant'. Specifically, we could test 300 separate linear mixed effects models with (baseline-corrected) pupil size for one specific $10 \mathrm{~ms}$ sample as dependent variable, cue eccentricity as fixed effect, and participant as random effect. Doing so reveals $p<.05$ for the effect of cue eccentricity at 125 samples.

However, this approach clearly raises the issue of multiple comparisons: when conducting so many statistical tests, there is a high chance that some of these tests will give significant but bogus effects; using statistical terminology, there will be a high chance of spurious effects or false alarms or type I errors, or the family-wise error rate exceeds the intended alpha level. To complicate matters further, pupil-size data is "auto-correlated": it changes very little from one 10 ms sample to the next. Because of this auto-correlation, you should not correct $p$ values with traditional techniques that assume that observations are uncorrelated, such as Bonferroni 
correction. To mitigate the issue of multiple comparisons, some researchers, including ourselves, have used an additional criterion, such as that $p<.05$ should hold for at least 200 contiguous milliseconds (e.g. Mathôt et al., 2017; see also Hershman et al., 2022 for a similar approach using Bayes Factors). This improves the issue somewhat, but it is not a formal way to correct for multiple comparisons; therefore, we no longer recommend this.

Sophisticated techniques for analyzing auto-correlated time series have been developed for functional magnetic resonance imaging (fMRI), electroencephalography (EEG), and magnetoencephalography (MEG; Bowman et al., 2020; Bullmore et al., 1999; Kriegeskorte et al., 2009; Maris, 2004; Maris \& Oostenveld, 2007); and although the specifics differ, the general principles that have been developed in these fields also apply to pupil-size data. Here we will focus on three techniques: using a predetermined time window, cluster-based permutation testing, and — our preferred technique—cross-validation.

\section{Using a predetermined time window}

If you have a strong prediction about when an effect should arise, you can conduct a single statistical test on the mean pupil size during the interval during which you predict the effect to emerge.

For our example experiment, we could derive a prediction from previous experiments that found that a voluntary shift of covert visual attention towards a bright or dark surface affects from about $750 \mathrm{~ms}$ after cue onset until the end of the trial (Mathôt et al., 2013). Based on this, we might predict that the effect of attentional breadth on pupil size similarly arises from $750 \mathrm{~ms}$ after the onset of the cue, and persists until the end of trial. We could then determine the mean (baseline-corrected) pupil size during this interval for each trial, and conduct a linear mixed 
effects analysis with Mean Pupil Size as dependent variable, Cue Eccentricity (-1: near, 0: medium, 1: far) as fixed effect, and Participant as random effect. Using an alpha level of .05, this test shows an effect of Cue Eccentricity $(z=2.28, p=.023)$ such that—as predicted—pupil size increases with increasing attentional breadth. An appropriate visualization of this test could be a line or bar plot that displays mean baseline-corrected pupil size as a function of cue eccentricity, with error bars that show the standard error across all trials (Fig. 10).

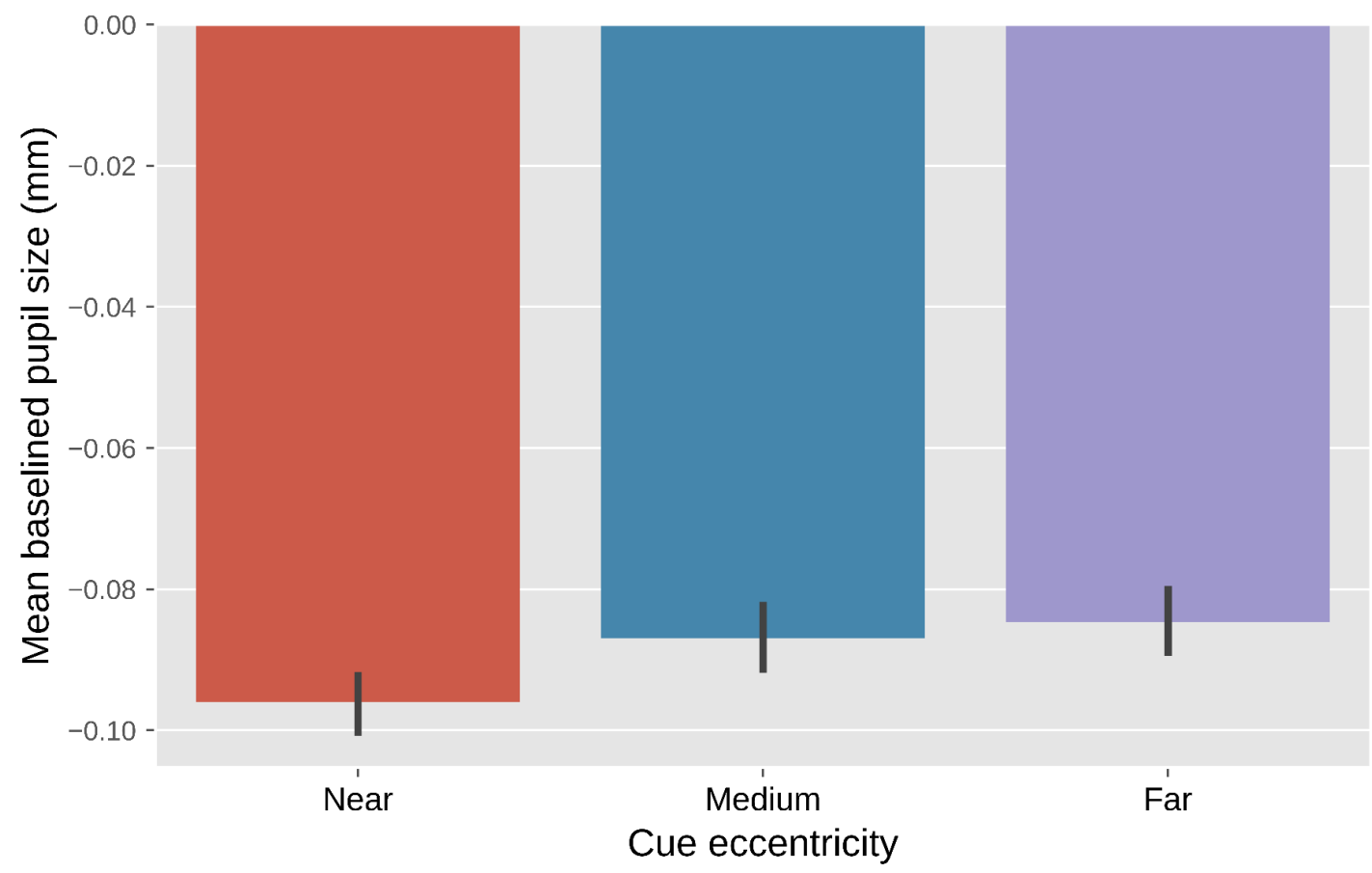

Figure 10. Results from our example experiment. Mean baselined pupil size during the $750 \mathrm{~ms}-3000 \mathrm{~ms}$ window after cue onset as a function of cue eccentricity. Values are negative due to an overall pupil constriction relative to the baseline period. Error bars indicate grand standard error.

The advantage of analyzing a predetermined time window is that there is no need to compensate for multiple corrections at all. The disadvantage is that, unless you are conducting a direct replication of a previous experiment, it is difficult to know in advance exactly at which point in 
time an effect, if it exists, will emerge: this depends on intra-individual variability, the stimulus display, task demands, and other factors (see also Bowman et al., 2020 for a similar point for EEG experiments).

\section{Cluster-based permutation testing}

Let's go back to the 300 separate tests that we conducted on the data from our example experiment. In this data, we found a cluster of 125 consecutive samples for which $p<.05$ (i.e. all 125 samples for which $p<.05$ comprised a single cluster). The crucial question is then, given that we are likely to find some samples for which $p<.05$, how likely are we to find a cluster of the size that we observed or larger, assuming that the null hypothesis is true? Phrased differently, what is the $p$ value of this cluster?

A cluster-based permutation test answers this question by randomly shuffling the condition labels on a trial basis and then running the same analysis again (Bullmore et al., 1999; Maris \& Oostenveld, 2007). Any cluster of samples for which $p<.05$ is then by necessity a false alarm. This procedure of shuffling and analyzing is repeated a large number of times (e.g. 1000 times), which results in a distribution of false-alarm clusters. The $p$ value for the actual cluster (i.e. the cluster size based on the unshuffled data) is then the proportion of false-alarm cluster sizes that are larger than or equal to the actual cluster size.

Cluster-based permutation tests are elegant and effective. However, they are not feasible when using linear mixed effects models to determine $p$ values for individual samples, because the time it would take to conduct the required number of tests is prohibitive. A single linear mixed effects model takes at least two seconds to run. To conduct a 1000 -fold cluster-based permutation test 
for a time series of 300 samples would take at least 1000 (permutations) $\times 300($ samples $) \times 2$ (seconds) $=600,000$ seconds, or one week (!) to compute.

The computational time required for a cluster-based permutation test can be vastly reduced by using a different underlying statistical technique, such as a repeated measures ANOVA, to determine $p$ values for individual samples. However, this would also result in decreased statistical power due to the fact that a repeated measures ANOVA is conducted on aggregated data (i.e. mean pupil size per participant and condition), whereas a linear mixed effects analysis is conducted on individual trials (Brysbaert \& Stevens, 2018). For this reason, we prefer to preserve the statistical power of linear mixed effects models, and combine this with cross-validation to control for multiple comparisons.

\section{Cross-validation testing}

Let's again go back to the 300 separate tests that we conducted on the data from our example experiment. Now that we have seen the data, we have a better idea where to look: the sample for which the $p$ value was lowest (sample $261, p=.002$ ). However, if we would actually do this, we would perform a circular analysis, in the sense that we would use the same data to both identify and test our result, which results in a very high chance of a false alarm (Bowman et al., 2020; Kriegeskorte et al., 2009).

Cross-validation is a general approach to avoid circularity in analyses by using one part of the data (the training set) to localize an effect, and another part of the data (the test set) to test the effect at the location that was identified in the training set. The Python library time_series_test ${ }^{4}$ implements cross-validation with linear mixed effects modeling in a

\footnotetext{
${ }^{4}$ https://github.com/smathot/time_series test
} 
way that is suitable for pupil-size data. Specifically, $75 \%$ of the data is used for the training set, and the remaining $25 \%$ of the data is used for the test set (using four-fold cross-validation, which is the default); the data is (by default) split in an interleaved fashion, such that three subsequent rows go into the training set, then the next row goes into the test set, the next three rows go into the training set, and so forth. A linear mixed effects model is then conducted for each sample of the training set. The sample that yields the highest $z$ value in the training set is used as the sample-to-be-tested for the test set. This procedure is repeated four times, using a different training set each time, until all samples have been part of a test set, and a sample-to-be tested has therefore been determined for the entire dataset. Finally, a single linear mixed effects model is conducted using the sample-to-be tested for each trial as a dependent measure. This means that the dependent variable consists of a column of (baseline-corrected) pupil-size values that correspond to different samples for different trials.

For models with multiple fixed effects, the cross-validation procedure is repeated for each main effect and interaction in the model. This is necessary because different effects may arise at different moments.

Using an alpha level of .05 , we ran a cross-validation analysis on our predetermined time-window of 750-3000 ms, and found an effect of Cue Eccentricity $(z=2.92, p=.004$, tested at samples 186 and 222) such that - as predicted - pupil size increases with increasing attentional breadth. An appropriate visualization of this test could be a 'trace plot' that shows mean pupil size as a function of time with cue eccentricities as separate lines (Fig. 11). The mean of the to-be-tested samples can then be shown as a vertical marker indicating when, approximately, the effect of cue eccentricity occurs most strongly, in this case at sample 204. 


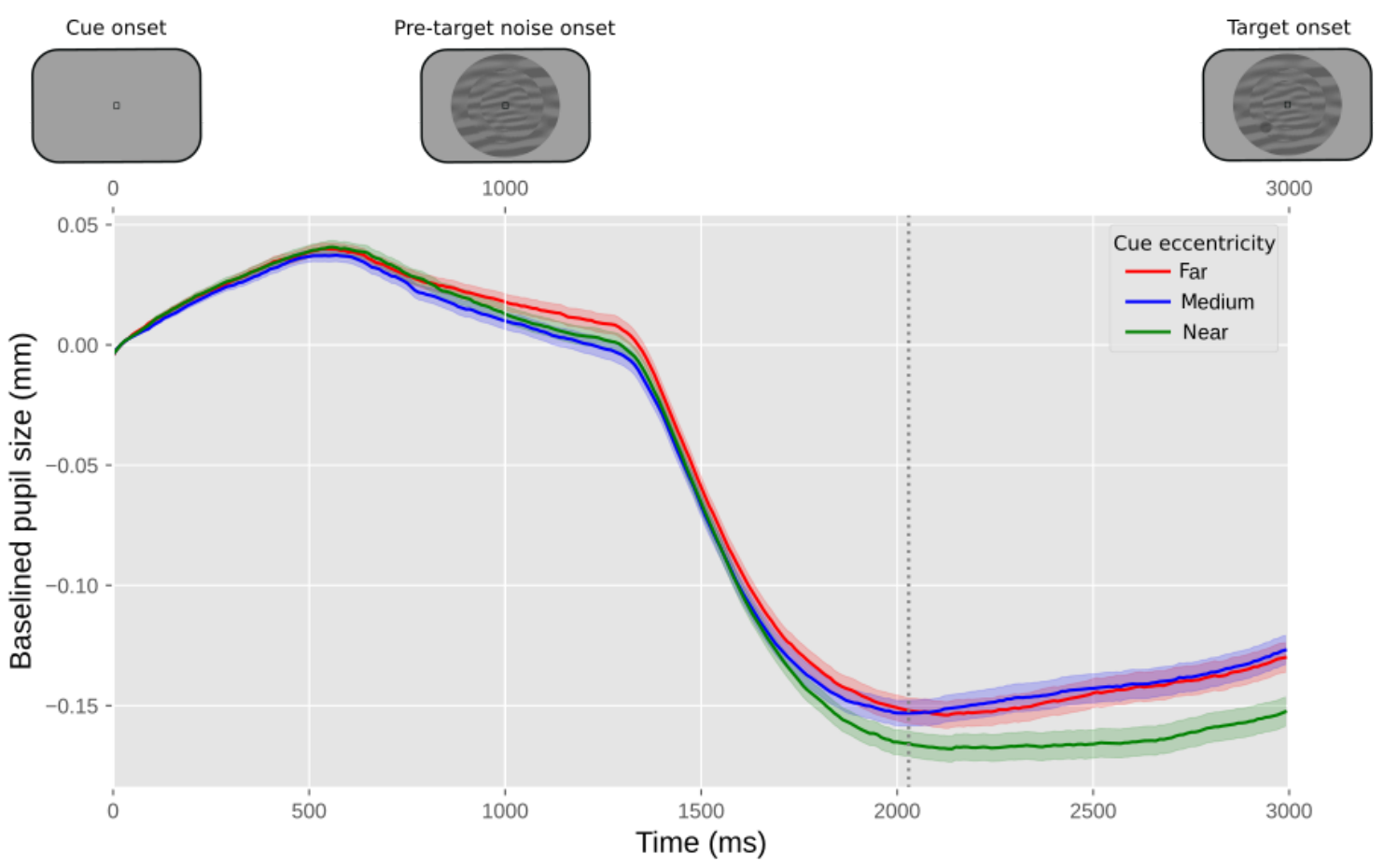

Figure 11. Results from our example experiment. The y-axis represents baseline-corrected pupil size. The lower $x$-axis represents time in milliseconds since cue onset. The upper $x$-axis represents the order of the events in the experiment. Differently colored lines represent the three cue eccentricities (far, medium, near). Error bands indicate grand standard error. The vertical line indicates the mean of the samples at which the effect of cue eccentricity was tested.

The main advantage of cross-validation, as compared to cluster-based permutation testing, is that it requires far fewer mixed effects models to run. For our example study, and again assuming at least two seconds of computational time for each linear mixed effects model, the test requires at least 4 (splits $) \times 300$ (samples $) \times 2$ (seconds $)=2,400$ seconds, or 40 minutes to compute. The test can be sped up considerably by using a so-called random-intercept only model for the localization phase (which is the default), and include by-participant random slopes, which is recommended by many statisticians (Barr et al., 2013), only for the final model that gives the final $z$ and $p$ values. For our example data and our test system, this reduces the computational 
time to about 5 minutes. Another advantage of cross-validation is that the outcome is deterministic - unlike the outcome of cluster-based permutation testing — at least when using a deterministic 'interleaved' method of splitting the data into test and training sets (which the default); that is, you always get the exact same outcome for the same data and analysis parameters.

\section{Conclusion}

Here we have provided a comprehensive, hands-on guide to cognitive pupillometry, which is the general approach of using pupil size as a measure for various cognitive processes. The guidelines are summarized in Fig 1. We have focused on (to us) 'typical' cognitive-pupillometry experiments, which are trial-based experiments in which a task-evoked pupil response to a stimulus is the measure of interest. We have outlined six basic principles for designing such experiments (see Experimental design):

- Stimuli should ideally be constant between conditions ("the Hillyard principle")

- Eye position should ideally be constant between conditions

- Trials should ideally be slow-paced

- Pupil size should ideally be measured while participants do nothing

- Ambient lighting should ideally be intermediate and matched to display brightness

- All data should ideally be stored in a single data file per participant

We further provided crucial steps for preprocessing pupil-size data, where 'preprocessing' refers to all steps involved in transforming raw data, as recorded during the experiment, into a format that is suitable for statistical analysis and visualization (see Preprocessing). We have also 
provided example Python code that illustrates these steps. In order (and the order matters!), the preprocessing steps are:

- Parsing raw data into an analysis-friendly data structure

- Interpolating or removing missing and invalid data (e.g. due to blinks)

- If necessary: downsampling

- If necessary: converting pupil size from arbitrary units to millimeters of diameter

- Baseline correction

- Verifying and visualizing data quality

- Excluding trials based on baseline pupil size

- If necessary: excluding participants based on data quality

Finally, we have discussed how to conduct statistical tests on pupil-size data (see Visualization and statistical analysis), highlighting the issue of multiple comparisons, which arises because pupil size consists of multiple observations over time, and it is often not known in advance at which time point an effect is expected to emerge. We have suggested three appropriate statistical approaches: conducting a single test on a predetermined time window; conducting a cluster-based permutation test; and conducting a cross-validation test. We have suggested that cross-validation is in many cases the preferred approach, because it does not require a time window to be determined a priori, and (unlike a cluster-based permutation test) it can be combined with linear mixed effects modeling without becoming prohibitively computationally intensive.

Finally, we have emphasized that our guidelines are illustrative rather than prescriptive: they are intended to provide researchers with a solid starting point for conducting cognitive-pupillometry experiments. 


\section{Open practices statement}

Experimental data and analysis scripts for this methods article are publicly accessible at https://osf.io/659pm/.

\section{References}

Barbur, J. L., Harlow, A. J., \& Sahraie, A. (1992). Pupillary responses to stimulus structure, colour and movement. Ophthalmic and Physiological Optics, 12(2), 137-141. https://doi.org/10.1111/j.1475-1313.1992.tb00276.x

Barr, D. J., Levy, R., Scheepers, C., \& Tily, H. J. (2013). Random effects structure for confirmatory hypothesis testing: Keep it maximal. Journal of Memory and Language, 68(3), 255-278. https://doi.org/10.1016/j.jml.2012.11.001

Beatty, J., \& Lucero-Wagoner, B. (2000). The pupillary system. In J. T. Cacioppo, L. G. Tassinary, \& G. G. Berntson (Eds.), Handbook of Psychophysiology (Vol. 2, pp. 142-162). Cambridge University Press.

Binda, P., \& Murray, S. O. (2015). Spatial attention increases the pupillary response to light changes. Journal of Vision, 15(2), 1. https://doi.org/10.1167/15.2.1

Binda, P., Pereverzeva, M., \& Murray, S. O. (2013a). Attention to bright surfaces enhances the pupillary light reflex. Journal of Neuroscience, 33(5), 2199-2204. https://doi.org/10.1523/JNEUROSCI.3440-12.2013

Binda, P., Pereverzeva, M., \& Murray, S. O. (2013b). Pupil constrictions to photographs of the sun. Journal of Vision, 13(6), e8. https://doi.org/10.1167/13.6.8

Bowman, H., Brooks, J. L., Hajilou, O., Zoumpoulaki, A., \& Litvak, V. (2020). Breaking the circularity in circular analyses: Simulations and formal treatment of the flattened average 
approach. PLOS Computational Biology, 16(11), e1008286.

https://doi.org/10.1371/journal.pcbi.1008286

Bradley, M. M., Miccoli, L., Escrig, M. A., \& Lang, P. J. (2008). The pupil as a measure of emotional arousal and autonomic activation. Psychophysiology, 45(4), 602-607. https://doi.org/10.1111/j.1469-8986.2008.00654.x

Bradshaw, J. L. (1969). Background light intensity and the pupillary response in a reaction time task. Psychonomic Science, 14(6), 271-272. https://doi.org/10.3758/BF03329118

Brisson, J., Mainville, M., Mailloux, D., Beaulieu, C., Serres, J., \& Sirois, S. (2013). Pupil diameter measurement errors as a function of gaze direction in corneal reflection eyetrackers. Behavior Research Methods, 45(4), 1322-1331. https://doi.org/10.3758/s13428-013-0327-0

Brocher, A., Harbecke, R., Graf, T., Memmert, D., \& Hüttermann, S. (2018). Using task effort and pupil size to track covert shifts of visual attention independently of a pupillary light reflex. Behavior Research Methods. https://doi.org/10.3758/s13428-018-1033-8

Brysbaert, M., \& Stevens, M. (2018). Power analysis and effect size in mixed effects models: A tutorial. Journal of Cognition, 1(1), 9. https://doi.org/10.5334/joc.10

Bullmore, E. T., Suckling, J., Overmeyer, S., Rabe-Hesketh, S., Taylor, E., \& Brammer, M. J. (1999). Global, voxel, and cluster tests, by theory and permutation, for a difference between two groups of structural MR images of the brain. IEEE Transactions on Medical Imaging, 18(1), 32-42. https://doi.org/10.1109/42.750253

Cherng, Y.-G., Baird, T., Chen, J.-T., \& Wang, C.-A. (2020). Background luminance effects on pupil size associated with emotion and saccade preparation. Scientific Reports, 10(1), $1-11$. 
Crawford, B. H. (1936). The dependence of pupil size upon external light stimulus under static and variable conditions. Proceedings of the Royal Society of London. Series B, Biological Sciences, 376-395. https://doi.org/10.1098/rspb.1936.0072

Dalmaijer, E., Mathôt, S., \& Van der Stigchel, S. (2014). PyGaze: An open-source, cross-platform toolbox for minimal-effort programming of eyetracking experiments. Behavior Research Methods, 46(4), 913-921. https://doi.org/10.3758/s13428-013-0422-2

Daniels, L. B., Nichols, D. F., Seifert, M. S., \& Hock, H. S. (2012). Changes in pupil diameter entrained by cortically initiated changes in attention. Visual Neuroscience, 29(02), 131-142. https://doi.org/10.1017/S0952523812000077

Einhäuser, W., Koch, C., \& Carter, O. L. (2010). Pupil dilation betrays the timing of decisions. Frontiers in Human Neuroscience, 4. https://doi.org/10.3389/fnhum.2010.00018

Fink, L., Simola, J., Tavano, A., Lange, E. B., Wallot, S., \& Laeng, B. (2021). From pre-processing to advanced dynamic modeling of pupil data. PsyArXiv. https://doi.org/10.31234/osf.io/wqvue

Gagl, B., Hawelka, S., \& Hutzler, F. (2011). Systematic influence of gaze position on pupil size measurement: Analysis and correction. Behavior Research Methods, 43(4), 1171-1181. https://doi.org/10.3758/s13428-011-0109-5

Geacintov, T., \& Peavler, W. S. (1974). Pupillography in industrial fatigue assessment. Journal of Applied Psychology, 59(2), 213-216. https://doi.org/10.1037/h0036529

Geller, J., Winn, M. B., Mahr, T., \& Mirman, D. (2020). GazeR: A package for processing gaze position and pupil size data. Behavior Research Methods, 52(5), 2232-2255. https://doi.org/10.3758/s13428-020-01374-8

Hayes, T. R., \& Petrov, A. A. (2016). Mapping and correcting the influence of gaze position on 
pupil size measurements. Behavior Research Methods, 48(2), 510-527.

https://doi.org/10.3758/s13428-015-0588-x

Hershman, R., Henik, A., \& Cohen, N. (2018). A novel blink detection method based on pupillometry noise. Behavior Research Methods, 50(1), 107-114. https://doi.org/10.3758/s13428-017-1008-1

Hershman, R., Henik, A., \& Cohen, N. (2019). CHAP: Open-source software for processing and analyzing pupillometry data. Behavior Research Methods, 51(3), 1059-1074. https://doi.org/10.3758/s13428-018-01190-1

Hershman, R., Milshtein, D., \& Henik, A. (2022). The contribution of temporal analysis of pupillometry measurements to cognitive research. Psychological Research. https://doi.org/10.1007/s00426-022-01656-0

Hess, E. H., \& Polt, J. M. (1960). Pupil size as related to interest value of visual stimuli. Science, 132(3423), 349-350. https://doi.org/10.1126/science.132.3423.349

Hess, E. H., \& Polt, J. M. (1964). Pupil size in relation to mental activity during simple problem-solving. Science, 143(3611), 1190-1192. https://doi.org/10.1126/science.143.3611.1190

Hoeks, B., \& Levelt, W. J. (1993). Pupillary dilation as a measure of attention: A quantitative system analysis. Behavior Research Methods, Instruments, \& Computers, 25(1), 16-26. https://doi.org/10.3758/BF03204445

Hong, S., Narkiewicz, J., \& Kardon, R. H. (2001). Comparison of pupil perimetry and visual perimetry in normal eyes: Decibel sensitivity and variability. Investigative Ophthalmology \& Visual Science, 42(5), 957-965.

Husta, C., Dalmaijer, E., Belopolsky, A., \& Mathot, S. (2019). The pupillary light response 
reflects visual working memory content. Journal of Experimental Psychology: Human Perception and Performance, 45(11), 1522. https://doi.org/10.1037/xhp0000689

Kahneman, D., \& Beatty, J. (1966). Pupil diameter and load on memory. Science, 154(3756), 1583-1585. https://doi.org/10.1126/science.154.3756.1583

Kelbsch, C., Strasser, T., Chen, Y., Feigl, B., Gamlin, P. D., Kardon, R., Peters, T., Roecklein, K. A., Steinhauer, S. R., Szabadi, E., Zele, A. J., Wilhelm, H., \& Wilhelm, B. J. (2019). Standards in Pupillography. Frontiers in Neurology, 10, 129. https://doi.org/10.3389/fneur.2019.00129

Kingdom, F. A. A., \& Prins, N. (2016). Psychophysics: A Practical Introduction. Academic Press.

Kinley, I., \& Levy, Y. (2021). PuPl: An open-source tool for processing pupillometry data. Behavior Research Methods, 1-24. https://doi.org/10.3758/s13428-021-01717-z

Knapen, T., Gee, J. W. de, Brascamp, J., Nuiten, S., Hoppenbrouwers, S., \& Theeuwes, J. (2016). Cognitive and ocular factors jointly determine pupil responses under equiluminance. PLOS ONE, 11(5), e0155574. https://doi.org/10.1371/journal.pone.0155574

Koelewijn, T., Zekveld, A. A., Festen, J. M., \& Kramer, S. E. (2012). Pupil dilation uncovers extra listening effort in the presence of a single-talker masker. Ear and Hearing, 33(2), 291-300. https://doi.org/10.1097/AUD.0b013e3182310019

Kret, M. E., \& Sjak-Shie, E. E. (2018). Preprocessing pupil size data: Guidelines and code. Behavior Research Methods, 1-7. https://doi.org/10.3758/s13428-018-1075-y

Kriegeskorte, N., Simmons, W. K., Bellgowan, P. S. F., \& Baker, C. I. (2009). Circular analysis in systems neuroscience: The dangers of double dipping. Nature Neuroscience, 12(5), 535-540. https://doi.org/10.1038/nn.2303 
Laeng, B., \& Sulutvedt, U. (2014). The eye pupil adjusts to imaginary light. Psychological Science, 25(1), 188-197. https://doi.org/10.1177/0956797613503556

Loewenfeld, I. E. (1958). Mechanisms of reflex dilatation of the pupil. Documenta Ophthalmologica, 12(1), 185-448. https://doi.org/10.1007/BF00913471

Lowenstein, O., Feinberg, R., \& Loewenfeld, I. E. (1963). Pupillary movements during acute and chronic fatigue: A new test for the objective evaluation of tiredness. Investigative Ophthalmology \& Visual Science, 2(2), 138-157.

Luck, S. J. (2005). Ten simple rules for designing ERP experiments. In T. C. Handy (Ed.), Event-related potentials: A methods handbook (Vol. 262083337). MIT Press.

Maris, E. (2004). Randomization tests for ERP topographies and whole spatiotemporal data matrices. Psychophysiology, 41(1), 142-151. https://doi.org/10.1111/j.1469-8986.2003.00139.x

Maris, E., \& Oostenveld, R. (2007). Nonparametric statistical testing of EEG- and MEG-data. Journal of Neuroscience Methods, 164(1), 177-190. https://doi.org/10.1016/j.jneumeth.2007.03.024

Markwell, E. L., Feigl, B., \& Zele, A. J. (2010). Intrinsically photosensitive melanopsin retinal ganglion cell contributions to the pupillary light reflex and circadian rhythm. Clinical and Experimental Optometry, 93(3), 137-149. https://doi.org/10.1111/j.1444-0938.2010.00479.x

Mathôt, S. (2013). A Simple Way to Reconstruct Pupil Size During Eye Blinks. http://dx.doi.org/10.6084/m9.figshare.688001

Mathôt, S. (2018). Pupillometry: Psychology, physiology, and function. Journal of Cognition, 1(1), 1-16. https://doi.org/10.5334/joc.18 
Mathôt, S., Dalmaijer, E., Grainger, J., \& Van der Stigchel, S. (2014). The pupillary light response reflects exogenous attention and inhibition of return. Journal of Vision, 14(14), 7. https://doi.org/10.1167/14.14.7

Mathôt, S., Fabius, J., Heusden, E. V., \& Stigchel, S. V. der. (2018). Safe and sensible preprocessing and baseline correction of pupil-size data. Behavior Research Methods, 1-13. https://doi.org/10.3758/s13428-017-1007-2

Mathôt, S., Grainger, J., \& Strijkers, K. (2017). Pupillary responses to words that convey a sense of brightness or darkness. Psychological Science, 28(8), 1116-1124. https://doi.org/10.1177/0956797617702699

Mathôt, S., \& Ivanov, Y. (2019). The effect of pupil size and peripheral brightness on detection and discrimination performance. PeerJ, 7, e8220. https://doi.org/10.7717/peerj.8220

Mathôt, S., Melmi, J. B., \& Castet, E. (2015). Intrasaccadic perception triggers pupillary constriction. PeerJ, 3(e1150), 1-16. https://doi.org/10.7717/peerj.1150

Mathôt, S., Schreij, D., \& Theeuwes, J. (2012). OpenSesame: An open-source, graphical experiment builder for the social sciences. Behavior Research Methods, 44(2), 314-324. https://doi.org/10.3758/s13428-011-0168-7

Mathôt, S., Siebold, A., Donk, M., \& Vitu, F. (2015). Large pupils predict goal-driven eye movements. Journal of Experimental Psychology: General, 144(3), 513-521. https://doi.org/10.1037/a0039168

Mathôt, S., van der Linden, L., Grainger, J., \& Vitu, F. (2013). The pupillary response to light reflects the focus of covert visual attention. PLoS ONE, 8(10), e78168. https://doi.org/10.1371/journal.pone.0078168

Naber, M., Alvarez, G. A., \& Nakayama, K. (2013). Tracking the allocation of attention using 
human pupillary oscillations. Frontiers in Psychology, 4(919), 1-12.

https://doi.org/10.3389/fpsyg.2013.00919

Naber, M., \& Nakayama, K. (2013). Pupil responses to high-level image content. Journal of Vision, 13(6), e7. https://doi.org/10.1167/13.6.7

Olmos-Solis, K., van Loon, A. M., \& Olivers, C. N. L. (2018). Pupil dilation reflects attentional priority settings prior to search. Journal of Cognition, 1(1), 11. https://doi.org/10.5334/joc. 12

Pan, J., Klímová, M., McGuire, J. T., \& Ling, S. (2022). Arousal-based pupil modulation is dictated by luminance. Scientific Reports, 12(1), 1390. https://doi.org/10.1038/s41598-022-05280-1

Petersch, B., \& Dierkes, K. (2021). Gaze-angle dependency of pupil-size measurements in head-mounted eye tracking. Behavior Research Methods. https://doi.org/10.3758/s13428-021-01657-8

Peysakhovich, V., Vachon, F., \& Dehais, F. (2017). The impact of luminance on tonic and phasic pupillary responses to sustained cognitive load. International Journal of Psychophysiology, 112, 40-45. https://doi.org/10.1016/j.ijpsycho.2016.12.003

Reilly, J., Kelly, A., Kim, S. H., Jett, S., \& Zuckerman, B. (2019). The human task-evoked pupillary response function is linear: Implications for baseline response scaling in pupillometry. Behavior Research Methods, 51(2), 865-878. https://doi.org/10.3758/s13428-018-1134-4

Reilly, J., Zuckerman, B., \& Kelly, A. (2021). A Primer on Design and Data Analysis for Cognitive Pupillometry. PsyArXiv. https://doi.org/10.31234/osf.io/j6sdt

Reimer, J., Froudarakis, E., Cadwell, C. R., Yatsenko, D., Denfield, G. H., \& Tolias, A. S. 
(2014). Pupil fluctuations track fast switching of cortical states during quiet wakefulness.

Neuron, 84(2), 355-362. https://doi.org/10.1016/j.neuron.2014.09.033

Rieger, G., Cash, B. M., Merrill, S. M., Jones-Rounds, J., Dharmavaram, S. M., \&

Savin-Williams, R. C. (2015). Sexual arousal: The correspondence of eyes and genitals.

Biological Psychology, 104, 56-64. https://doi.org/10.1016/j.biopsycho.2014.11.009

Slooter, J. H., \& van Norren, D. (1980). Visual acuity measured with pupil responses to checkerboard stimuli. Investigative Ophthalmology \& Visual Science, 19(1), 105-108.

Snell, J., Mathôt, S., Mirault, J., \& Grainger, J. (2018). Parallel graded attention in reading: A pupillometric study. Scientific Reports, 8(1), 3743.

https://doi.org/10.1038/s41598-018-22138-7

Steinhauer, S. R., Siegle, G. J., Condray, R., \& Pless, M. (2004). Sympathetic and parasympathetic innervation of pupillary dilation during sustained processing. International Journal of Psychophysiology, 52(1), 77-86. https://doi.org/10.1016/j.ijpsycho.2003.12.005

Stoll, J., Chatelle, C., Carter, O., Koch, C., Laureys, S., \& Einhäuser, W. (2013). Pupil responses allow communication in locked-in syndrome patients. Current Biology, 23(15), R647-R648. https://doi.org/10.1016/j.cub.2013.06.011

Tyukhova, Y., \& Waters, C. (2019). Subjective and pupil responses to discomfort glare from small, high-luminance light sources. Lighting Research \& Technology, 51(4), 592-611. https://doi.org/10.1177/1477153518772000

Ukai, K. (1985). Spatial pattern as a stimulus to the pupillary system. Journal of the Optical Society of America A, 2(7), 1094-1100. https://doi.org/10.1364/JOSAA.2.001094 Unsworth, N., \& Robison, M. K. (2014). Individual differences in the allocation of attention to 
items in working memory: Evidence from pupillometry. Psychonomic Bulletin \& Review, 1-9. https://doi.org/10.3758/s13423-014-0747-6

Unsworth, N., \& Robison, M. K. (2017). Pupillary correlates of covert shifts of attention during working memory maintenance. Attention, Perception, \& Psychophysics, 79(3), 782-795. https://doi.org/10.3758/s13414-016-1272-7

Urai, A. E., Braun, A., \& Donner, T. H. (2017). Pupil-linked arousal is driven by decision uncertainty and alters serial choice bias. Nature Communications, 8(1), 14637. https://doi.org/10.1038/ncomms 14637

Van de Kraats, J., Smit, E. P., \& Slooter, J. H. (1977). Objective perimetric measurements by the pupil balance method. Second International Visual Field Symposium, Tubingen, 19-22 September, 1976, 213-220.

van Hooijdonk, R., Mathot, S., Schat, E., Spencer, H., van der Stigchel, S., \& Dijkerman, H. C. (2019). Touch-induced pupil size reflects stimulus intensity, not subjective pleasantness. Experimental Brain Research, 237(1), 201-210. https://doi.org/10.1007/s00221-018-5404-2

Wang, C.-A., \& Munoz, D. P. (2014). Modulation of stimulus contrast on the human pupil orienting response. European Journal of Neuroscience, 1-11. https://doi.org/10.1111/ejn.12641

Wang, C.-A., \& Munoz, D. P. (2015). A circuit for pupil orienting responses: Implications for cognitive modulation of pupil size. Current Opinion in Neurobiology, 33, 134-140. https://doi.org/10.1016/j.conb.2015.03.018

Wilschut, T., \& Mathot, S. (2022). Interactions between visual working memory, attention, and color categories: A pupillometry study. Journal of Cognition, 5(1), 16. 
https://doi.org/10.5334/joc. 208

Winn, M. B., Wendt, D., Koelewijn, T., \& Kuchinsky, S. E. (2018). Best practices and advice for using pupillometry to measure listening effort: An introduction for those who want to get started. Trends in Hearing, 22, 1-32. https://doi.org/10.1177/2331216518800869

Yoo, K., Ahn, J., \& Lee, S.-H. (2021). The confounding effects of eye blinking on pupillometry, and their remedy. PLOS ONE, 16(12), e0261463.

https://doi.org/10.1371/journal.pone.0261463

Zekveld, A. A., Kramer, S. E., \& Festen, J. M. (2010). Pupil response as an indication of effortful listening: The influence of sentence intelligibility. Ear and Hearing, 31(4), 480-490. https://doi.org/10.1097/AUD.0b013e3181d4f251

Zokaei, N., Board, A. G., Manohar, S. G., \& Nobre, A. C. (2019). Modulation of the pupillary response by the content of visual working memory. Proceedings of the National Academy of Sciences, 116(45), 22802-22810. https://doi.org/10.1073/pnas.1909959116

Zuber, B. L., Stark, L., \& Lorber, M. (1966). Saccadic suppression of the pupillary light reflex. Experimental Neurology, 14(3), 351-370. https://doi.org/10.1016/0014-4886(66)90087-2 